\title{
Enhanced Higgs mass in Compact Supersymmetry
}

\author{
Kohsaku Tobioka, ${ }^{a, b, c}$ Ryuichiro Kitano ${ }^{c, d, e}$ and Hitoshi Murayama ${ }^{e, f, g}$ \\ ${ }^{a}$ Raymond and Beverly Sackler School of Physics and Astronomy, Tel-Aviv University, \\ Tel-Aviv 69978, Israel \\ ${ }^{b}$ Department of Particle Physics and Astrophysics, Weizmann Institute of Science, \\ Herzl St 234, Rehovot 7610001, Israel \\ ${ }^{c}$ Theory Center, High Energy Accelerator Research Organization (KEK), \\ 1-1 Oho, Tsukuba 305-0801, Japan \\ ${ }^{d}$ The Graduate University for Advanced Studies (Sokendai), \\ 1-1 Oho, Tsukuba 305-0801, Japan \\ ${ }^{e}$ Kavli Institute for the Physics and Mathematics of the Universe (WPI), \\ University of Tokyo Institutes for Advanced Study, University of Tokyo, \\ 5-1-5 Kashiwanoha, Kashiwa 277-8583, Japan \\ ${ }^{f}$ Department of Physics, University of California, Berkeley, \\ 366 LeConte Hall, Berkeley, California 94720, U.S.A. \\ ${ }^{g}$ Theoretical Physics Group, Lawrence Berkeley National Laboratory, \\ 1 Cyclotron Rd, Berkeley, California 94720, U.S.A. \\ E-mail: tobioka@post.kek.jp, Ryuichiro.Kitano@kek.jp, \\ hitoshi.murayama@ipmu.jp
}

ABSTRACT: The current LHC results make weak scale supersymmetry difficult due to relatively heavy mass of the discovered Higgs boson and the null results of new particle searches. Geometrical supersymmetry breaking from extra dimensions, Scherk-Schwarz mechanism, is possible to accommodate such situations. A concrete example, the Compact Supersymmetry model, has a compressed spectrum ameliorating the LHC bounds and large mixing in the top and scalar top quark sector with $\left|A_{t}\right| \sim 2 m_{\tilde{t}}$ which radiatively raises the Higgs mass. While the zero mode contribution of the model has been considered, in this paper we calculate the Kaluza-Klein tower effect to the Higgs mass. Although such contributions are naively expected to be as small as a percent level for $10 \mathrm{TeV}$ Kaluza-Klein modes, we find the effect significantly enhances the radiative correction to the Higgs quartic coupling by from 10 to $50 \%$. This is mainly because the top quark wave function is pushed out from the brane, which makes the top mass depend on higher powers in the Higgs field. As a result the Higgs mass is enhanced up to $15 \mathrm{GeV}$ from the previous calculation. We also show the whole parameter space is testable at the LHC run II.

KEYWORDS: Supersymmetry Phenomenology

ArXiv EPrint: 1511.04081 


\section{Contents}

1 Introduction 1

2 Overview 3

2.1 Scherk-Schwartz mechanism and Compact Supersymmetry model 3

2.2 Picture of Radion Mediation 5

3 Effective potential $\quad 6$

$\begin{array}{lll}3.1 & \text { Effective potential } & 7\end{array}$

3.2 Enhancement of Higgs quartic coupling 8

4 Higgs mass $\quad 9$

$\begin{array}{lll}4.1 & \text { Electroweak symmetry breaking } & 10\end{array}$

$\begin{array}{lll}4.2 & \text { Higher order corrections } & 11\end{array}$

$\begin{array}{lll}4.3 & \text { Enhanced Higgs mass } & 11\end{array}$

5 Experimental bounds $\quad 13$

$\begin{array}{llr}6 & \text { Conclusions } & 14\end{array}$

A Mass spectrum $\quad \mathbf{1 5}$

$\begin{array}{ll}\text { A.1 Equations of motion } & 15\end{array}$

A.2 Solution for quark 16

$\begin{array}{ll}\text { A.3 Solutions for squarks } & 18\end{array}$

$\begin{array}{lr}\text { B Infinite sum } & 20\end{array}$

C Effect of higher order terms of $\mathcal{O}\left(H_{u}^{6} R^{6}\right)$ to Higgs Mass 22

D Threshold corrections to Higgs soft terms 24

\section{Introduction}

Supersymmetry is the prime candidate for the physics beyond the standard model (for a review [1]). It can stabilizes the large hierarchy between the electroweak scale and some high energy scale such as the quantum gravity scale, and also it leads to dynamical electroweak symmetry breaking by radiative correction effects. The minimal supersymmetric standard model (MSSM) has been studied as an attractive and minimal model of supersymmetry. However under its constrained framework, the lightest Higgs mass has an upper bound of $m_{Z} \simeq 91 \mathrm{GeV}$ at tree level, and hence this has a very strong tension with the recently 
discovered Higgs boson at the Large Hadron Collider (LHC) [2, 3]. As the latest result, the combined analysis by the ATLAS and CMS says that the measured mass is $125.09 \pm$ $0.21 \pm 0.11 \mathrm{GeV}$ [4]. To explain the discrepancy it is known that the Higgs mass can be raised beyond $m_{Z}$ by radiative corrections through large scalar top (stop) mass or large mixing between left and right stops (large $A$-term) but in most cases fine-tuning of less than percent level is accompanied with those radiative corrections [5]. Various extensions to the minimal model are also considered in order to accommodate the Higgs mass. One direction is to introduce singlet(s) to the Higgs sector resulting in non-decoupling $F$-term as in NMSSM [6-9] and Dirac-NMSSM [10], or to introduce an extra gauge group under which Higgs is charged resulting in non-decoupling $D$-term $[11,12]$. Also a strongly coupled Higgs sector is another possibility [13-15].

On the other hand, supersymmetric particles (sparticles) has been extensively searched for at the LHC. So far the null result has been found at the LHC run I and therefore the strong constraints on the parameter space are obtained. Typical supersymmetric models such as the Constrained MSSM (CMSSM) [16] has an exclusion bound on sparticles mass beyond $\mathrm{TeV}$ [17], which is another tension in addition to the discrepancy of the Higgs mass. The sparticle searches are mainly based on missing transverse energy, $\mathbb{E}_{T},{ }^{1}$ motivated by $R$ parity, and the corresponding exclusion bounds are often strong but still model dependent. The signal becomes weaker, even in presence of enouch sparticle production, due to small missing energy when a mass spectrum is compressed $[18,19]$ or sparticles decay to new states $[20,21]$, or due to lack of missing energy signal when $R$-parity is violated [22-26].

The Compact Supersymmetry model has a possibility to accommodate those tensions because it has a compressed spectrum and large $A$-term [19]. The model is embedded in $5 \mathrm{D}$ spacetime with a simple extra dimension, $S^{1} / \mathbb{Z}_{2}$, and has a field configuration that quark, lepton and gauge superfields are in the bulk while Higgs fields are localized on a brane. Supersymmetry is broken by non-trivial boundary conditions of the extra dimension, called the Scherk-Schwartz mechanism [27, 28]. As a direct consequence of the field configuration and the Scherk-Schwartz mechanism, the universal soft masses, which is important for a compressed spectrum, and near maximal mixing by $A$-term $\left(\left|A_{t}\right| \sim 2 m_{\tilde{t}}\right)$, which enhances the Higgs mass beyond $m_{Z}$, are obtained. Requirement of the successful electroweak symmetry breaking fixes the supersymmetric Higgs mass, $\mu$ term, which determines the mass scale of the lightest sparticle (LSP). As a result, a spectrum with mass compression of a few hundreds of $\mathrm{GeV}$ is realized in generic parameter space ameliorating the LHC bounds. The model has further attractive features. The geometrical nature of supersymmetry breaking does not introduce the conventional $\mathrm{CP}$ or flavor problems, and there are only three free parameters two of which are left after requiring the successful electroweak symmetry breaking.

Regarding the Higgs mass, the previous computation for the model has included the only zero mode contributions, that is the MSSM contribution, and the Higgs mass was

\footnotetext{
${ }^{1}$ To be precise, this should be called as missing transverse momentum because we can only measure a missing quantity constructed by transverse momentum conservation, $\overrightarrow{\boldsymbol{P}}_{T}=-\sum \vec{P}_{T}^{\text {vis }}$. In fact, in a scenario of compressed spectrum, the energy carried out of the detector is large but the missing momentum can be small. However here we use the convention that missing transverse momentum is called as $\mathbb{E}_{T}$.
} 
expected to be 119 to $125 \mathrm{GeV}$ even for TeV sparticle thanks to the large $A$-term. Interestingly, without adding any extra things such as singlets, it can be further enhanced by a contribution from the Kaluza-Klein (KK) tower. This possibility is implied by the Constrained Standard model [29] which is another model with the Scherk-Schwarz mechanism and has a similar Higgs sector of the Compact Supersymmetry model. It is pointed out in ref. [29] that the KK tower drastically changes the Higgs potential and the Higgs mass is highly enhanced even with very light stop. While the Constrained Standard model is not compatible with the LHC results, the KK effect remains interesting especially because the observed Higgs mass of $125 \mathrm{GeV}$ is relatively heavy for the MSSM. In this paper we revisit the effect of KK tower to the Higgs sector with general supersymmetry breaking parameter because the Constrained Standard model focused on the maximal breaking case. Then we apply the result to the Higgs mass calculation. We find the effect remains large even when the KK modes are at $\mathcal{O}(10) \mathrm{TeV}$, and the enhancement of the Higgs mass from the MSSM calculation is from 5 to $15 \mathrm{GeV}$ in interesting parameter regions. Since $125 \mathrm{GeV}$ Higgs mass is realized in a lower supersymmetry breaking scale, $\mathrm{TeV}$ range of sparticle mass is still compatible with the observed Higgs mass and the LHC sparticle searches in this model. An interesting upper bound of sparticle mass is obtained by the dark matter relic density, which implies that the whole parameter space can be tested by the LHC run II.

The Scherk-Schwarz mechanism has been discussed with a special attention to the UV-finite feature: not only quadratic divergence is absent but also log divergence is absent [29-39]. This is because of the non-local nature of supersymmetry breaking. We see such a feature in our calculation of the radiative corrections from the KK tower. Also many models beyond the Standard Model with various field configurations are discussed in refs. [29-32, 34, 36, 38, 40-49]. As a further extension, the mechanism is used in Folded Supersymmetry models $[50,51]$.

In the following section we present an overview of the Scherk-Schwartz mechanism and the Compact Supersymmetry model. We compute radiative corrections of the KK tower to the Higgs sector in section 3 and apply the result to the Higgs mass calculation in section 4. In section 5. we study experimental bounds and show the LHC bound is certainly weaker due to the compressed spectrum. We conclude in section 6 .

\section{Overview}

\subsection{Scherk-Schwartz mechanism and Compact Supersymmetry model}

We consider a single compact extra dimension with its coordinate $y$ identified by translation, $\mathcal{T}: y \rightarrow y+2 \pi R$, and reflection, $\mathcal{Z}: y \rightarrow-y$, where $R$ is the radius of the extra dimension. This is $S^{1} / \mathbb{Z}_{2}$ orbifold, and it is subject to two consistency conditions,

$$
\mathcal{P}^{2}=1, \quad \mathcal{P} \mathcal{T} \mathcal{P}=\mathcal{T}^{-1}
$$

The minimum supersymmetry in $5 \mathrm{D}$ corresponds to $\mathcal{N}=2$ in $4 \mathrm{D}$, leading to a global $\mathrm{SU}(2)_{R}$ symmetry. In presence of such a global symmetry, $\mathrm{SU}(2)_{R}$ doublets, such as gaug- 


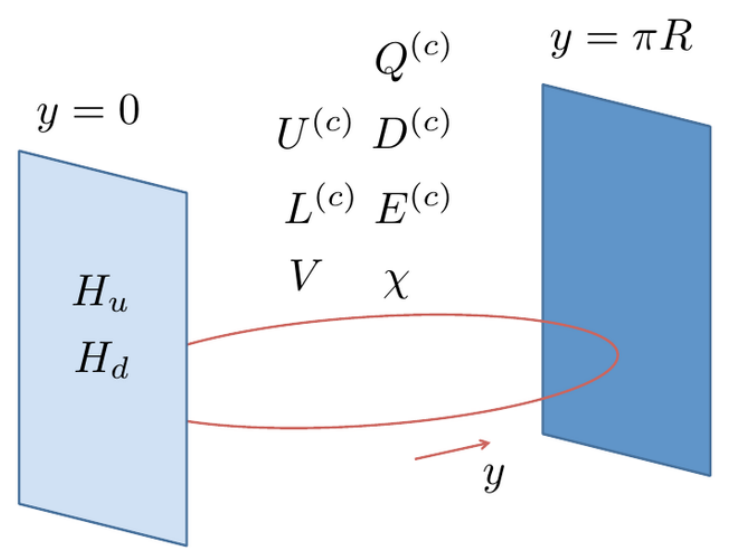

Figure 1. Field configurations of the Compact Supersymmetry model. Hypermultiplets of quark and lepton and gauge fields are living in the whole 5D spacetime while Higgs chiral superfields are localized on a brane.

inos, can have non-trivial boundary conditions with a twist of $\alpha$ under the translation,

$$
\mathcal{P}=\left(\begin{array}{cc}
1 & 0 \\
0 & -1
\end{array}\right), \quad \mathcal{T}=e^{(2 \pi \alpha) i \sigma_{2}}=\left(\begin{array}{cc}
\cos (2 \pi \alpha) & \sin (2 \pi \alpha) \\
-\sin (2 \pi \alpha) & \cos (2 \pi \alpha)
\end{array}\right),
$$

where $0 \leq \alpha \leq 1 / 2$. We are interested in $\alpha \ll 1$. On the other hand, $\mathrm{SU}(2)_{R}$ singlets have only trivial boundary conditions of $\mathcal{P}= \pm 1$ and $\mathcal{T}=1$ because they do not have a matrix structure. The finite twist of $\alpha$ leads to mass splitting in supermultiplets and then supersymmetry is broken. This mechanism is called the Scherk-Schwarz mechanism [27, 28].

The Scherk-Schwarz mechanism is applied in the Compact Supersymmetry model [19] in which three-generation of quarks and leptons as well as the Standard Model gauge groups are embedded in a bulk (extra dimension) and two Higgs fields, $H_{u}$ and $H_{d}$, are localized on a brane at $y=0$. A schematic picture of the model seen in figure 1. A bulk field forms a hypermultiplet that have two chiral superfields. One of them, $\Phi=Q, U, D, L, E$, has a reflection property of $\mathcal{P}=1$ and keeps zero mode. The other chiral superfield is denoted with a superscript as $\Phi^{c}$ and has a property of $\mathcal{P}=-1$ leading to absence of zero mode. Under the translation, the squark (slepton) in $\Phi$ mixes with the squark (slepton) in $\Phi^{c}$ with the same twist as in eq. (2.2) because squarks (sleptons) are $\mathrm{SU}(2)_{R}$ doublets. Since the twist parameter is unique in a single global symmetry, $\mathrm{SU}(2)_{R}$ doublets, gauginos, squarks, sleptons and gravitinos, must have the same soft mass of $\alpha / R$ after the KK expansion. A similar model where Higgs lives in the bulk as well as matters is studied in ref. [43].

Using $\mathcal{P}=1$ chiral superfields, we can write Yukawa couplings and $\mu$ term,

$$
\mathcal{W}_{\text {brane }}=\delta(y)\left\{y_{U 5} H_{u} Q U+y_{D 5} H_{d} Q D+y_{L 5} H_{d} L E+\mu H_{u} H_{d}\right\} \text {. }
$$

Matter fields have a non-trivial twist by the Scherk-Schwarz mechanism giving trilinear scalar couplings which corresponds to soft supersymmetry breaking of Yukawa couplings, $A$-terms. However, the Higgs are just 4D field and do not feel supersymmetry breaking at 
the tree level, and therefore soft terms related only to Higgs are absent. In summary, using the conventional MSSM notation, the soft breaking terms are given by

$$
M_{1 / 2}=\frac{\alpha}{R}, \quad m_{\tilde{Q}, \tilde{U}, \tilde{D}, \tilde{L}, \tilde{E}}^{2}=\frac{\alpha^{2}}{R^{2}}, \quad A_{0}=-\frac{2 \alpha}{R}, \quad m_{H_{u}, H_{d}}^{2}=0, \quad b=0 .
$$

This is more easily derived in an equivalent picture of the Radion Mediation in section 2.2. We emphasize that the model realizes the large $A$-term, $A_{0} \approx-2 m_{\tilde{t}}$, which enhances the lightest Higgs mass and may explain the observed value, and we will show that it is enhanced even beyond this expectation. It is noteworthy that the conventional supersymmetric CP and flavor problems are absent thanks to geometric nature of supersymmetry breaking. Then model has a compact parameter set of $\alpha, R$, and $\mu$ even more constrained than the CMSSM, which implies testability of the model. Note that ref. [48] addresses the same framework with the soft breaking of eq. (2.4) as a possible solution to the little hierarchy problem [52].

\section{$2.2 \quad$ Picture of Radion Mediation}

On $S^{1} / \mathbb{Z}_{2}$ orbifold, refs. [53,54] show that the Scherk-Schwarz mechanism is equivalent to the Radion Mediation [42]. The Radion Mediation gives supersymmetry breaking by the $F$-term vacuum expectation value of the Radion chiral superfield, $T$, and hence it is more comprehensive. The 5D bulk action based on the $4 \mathrm{D}$ superspace [55] is

$$
\begin{aligned}
\mathcal{K}_{5}= & \frac{T+T^{\dagger}}{2 R}\left\{\Phi^{\dagger} e^{-V} \Phi+\Phi^{c} e^{V} \Phi^{c \dagger}\right\} \\
& +\frac{1}{8 k g_{5}^{2}} \frac{2 R}{T+T^{\dagger}} \operatorname{Tr}\left[\left(\partial_{5}+\sqrt{2} \chi^{\dagger}\right) e^{-V}\left(-\partial_{5}+\sqrt{2} \chi\right) e^{V}+\frac{\partial_{5} e^{-V} \partial_{5} e^{V}}{2}+\left(\chi \chi+\chi^{\dagger} \chi^{\dagger}\right)\right] \\
\mathcal{W}_{5}= & \Phi^{c}\left(\partial_{5}-\sqrt{2} \chi\right) \Phi+\frac{1}{16 k g_{5}^{2}} \frac{T}{R} \operatorname{Tr}\left[W_{\alpha} W^{\alpha}\right]
\end{aligned}
$$

where $V$ is gauge superfield, $\chi$ is adjoint chiral superfield, and $\operatorname{Tr}\left[T^{a} T^{b}\right]=k \delta^{a b} . V$ and $\chi$ form a $5 \mathrm{D}$ vector supermultplet. Here, fields have trivial boundary conditions under translation, $\mathcal{T}=1$, but supersymmetry breaking is introduced by the Radion VEV,

$$
\langle T\rangle=R+\theta^{2} F_{T} .
$$

As shown in ref. [54], $F_{T}$ can be removed from the action if a twist is introduced in the global $\mathrm{SU}(2)_{R}$ space, resulting in a correspondence of $F_{T}=2 \alpha$. Then the gaugino mass is $M_{1 / 2}=F_{T} /(2 R)=\alpha / R$ from eq. (2.6).

It is easy to see the size of other soft terms. Since Kähler potential for matter fields is not canonically normalized, we normalize the bulk fields by shifting $F$ terms of matter fields such that

$$
\left\{Q^{(c)}, U^{(c)}, D^{(c)}, L^{(c)}, E^{(c)}\right\} \rightarrow\left(1-\frac{\alpha}{R} \theta^{2}\right)\left\{Q^{(c)}, U^{(c)}, D^{(c)}, L^{(c)}, E^{(c)}\right\} .
$$

The squark and slepton masses are given by residual $(\alpha / R)^{2} \theta^{2} \bar{\theta}^{2}$ term. Also, a Yukawa coupling comes up with a large $A$-term, for example, the field redefinition of eq. (2.8) leads to

$$
\int d^{2} \theta y_{U 5} H_{u} Q U \rightarrow \int d^{2} \theta\left(1-\frac{\alpha}{R} \theta^{2}\right)^{2} y_{U 5} H_{u} Q U=\int d^{2} \theta\left(1-\frac{2 \alpha}{R} \theta^{2}\right) y_{U 5} H_{u} Q U
$$


$A_{0}=-2 \alpha / R$ is shown. It is easy to see that this supersymmetry breaking has the minimal flavor violation structure. Therefore all the soft terms in the Radion Mediation matches with eq. (2.4).

\section{Effective potential}

We calculate the effective potential from 1-loop of all the KK modes of top quark and squark to take into account their effect to the lightest Higgs mass. The 5D Lagrangian for the up-type squark and quark bilinears is given by

$$
\begin{aligned}
\mathcal{L}_{5}= & \tilde{Q}^{\dagger}\left(\partial^{2}-\partial_{5}^{2}\right) \tilde{Q}+\tilde{Q}^{c \dagger}\left(\partial^{2}-\partial_{5}^{2}\right) \tilde{Q}^{c}+\tilde{U}^{\dagger}\left(\partial^{2}-\partial_{5}^{2}\right) \tilde{U}+\tilde{U}^{c \dagger}\left(\partial^{2}-\partial_{5}^{2}\right) \tilde{U}^{c} \\
& +\delta(y)\left(y_{U 5} H_{u} \tilde{Q} \partial_{5} \tilde{U}^{c *}+y_{U 5} H_{u} \tilde{U} \partial_{5} \tilde{Q}^{c *}+\text { h.c. }\right)-\left|\delta(y) y_{U 5} H_{u} \tilde{Q}\right|^{2}-\left|y_{U 5} H_{u} \tilde{U} \delta(y)\right|^{2} \\
& +\bar{\Psi}_{Q}\left(i \not \partial+\gamma_{5} \partial_{5}\right) \Psi_{Q}+\bar{\Psi}_{U}\left(i \not \partial+\gamma_{5} \partial_{5}\right) \Psi_{U}-\delta(y) y_{U 5} H_{u} \bar{\Psi}_{Q}^{c} P_{L} \Psi_{U}+\delta(y) y_{U 5}^{*} \bar{\Psi}_{U} P_{R} \Psi_{Q}^{c} H_{u}^{*},
\end{aligned}
$$

where $\tilde{Q}^{(c)}=\tilde{Q}^{(c)}(x, y), \tilde{U}^{(c)}=\tilde{U}^{(c)}(x, y), H_{u}=H_{u}(x)$, and $\Psi$ represents a 4-component fermion,

$$
\Psi_{Q} \equiv\left(\begin{array}{c}
Q(x, y) \\
\bar{Q}^{c}(x, y)
\end{array}\right), \quad \Psi_{U} \equiv\left(\begin{array}{c}
U(x, y) \\
\bar{U}^{c}(x, y)
\end{array}\right) .
$$

Before going to the effective potential, we have to obtain Higgs-dependent mass eigenvalues. Since it is difficult to obtain the mass spectrum after the KK expansion due to infinite mixing terms coming from $\delta(y)$, we derive mass eigenvalues by solving $5 \mathrm{D}$ equations of motion. We first generally solve Dirac and Klein-Gordon equations in the bulk respecting properties under the reflection, secondly constraint the solutions by the boundary condition of the translation (the Scherk-Schwarz mechanism), and finally determine the coefficients by integrating around the brane at $y=0$. The integration around the brane gives the Higgs field dependence to quark and squark masses. The detail is given in appendix A.

The above computation leads to consistency conditions of mass in eqs. (A.29), (A.51). One for top quark is

$$
\tan ^{2}\left(M_{F} \pi R\right)=\left(\frac{y_{t 5} H_{u}}{2}\right)^{2}
$$

and this gives mass eigenvalues of KK tower in presence of Higgs VEV,

$$
M_{F}=\frac{n}{R} \pm M_{t}\left(H_{u}\right) \quad(n: \text { integer }),
$$

where

$$
M_{t}\left(H_{u}\right) \equiv \frac{1}{\pi R} \arctan \left(\frac{y_{t 5} H_{u}}{2}\right)=y_{t} H_{u}-\frac{(\pi R)^{2}\left(y_{t} H_{u}\right)^{3}}{3}+\mathcal{O}\left(H_{u}^{5} R^{4}\right) .
$$

The top Yukawa coupling in $4 \mathrm{D}$ is given by $y_{t}=y_{t 5} / 2 \pi R$. Note that the top mass, $M_{t}$, is not only proportional to $H_{u}$ but also has higher powers of $H_{u}$. This can be understood as follows. The wave function of top quark zero mode is flat in absence of the Higgs VEV, but is distorted by the non-zero Higgs VEV at $y=0$. Especially, top quark tends to reduce overlap with Higgs to minimize the energy. This is why top mass has non-trivial 
dependence of $H_{u}$. In the language of $4 \mathrm{D}$ effective theory, the term of $\mathcal{O}\left(H_{u}^{3}\right)$ is due to higher dimensional operators generated by the non-zero KK modes and the coefficient actually could be explained by summation of the tower, $\sum_{n=1}^{\infty} \frac{1}{n^{2} / R^{2}}=R^{2} \zeta(2)=\pi^{2} R^{2} / 6$.

Similarly, the consistency condition for stop is

$$
\cos \left(2 \pi M_{B} R\right)=\cos \left(2 \pi \alpha \pm 2 \pi M_{t} R\right)
$$

which leads to mass eigenvalues,

$$
M_{B}=\frac{n+\alpha}{R} \pm M_{t}\left(H_{u}\right) \quad(n: \text { integer }) .
$$

This result makes sense because supersymmetric limit, $\alpha=0$, reproduces quark mass spectrum and also because mass splitting of zero mode squarks, $\pm M_{t}$, is as expected by $A_{0}=-2 \alpha / R$.

\subsection{Effective potential}

Once the Higgs-dependent mass spectrum is obtained, we can compute the effective potential,

$$
V_{t}=\frac{2 N_{c}}{2 R^{4}} \sum_{n=-\infty}^{\infty} \int \frac{d^{4} k}{(2 \pi)^{4}}\left\{\log \frac{k^{2}+\left(n+\omega_{B+}\right)^{2}}{k^{2}+\left(n+\omega_{F}\right)^{2}}+\log \frac{k^{2}+\left(n+\omega_{B-}\right)^{2}}{k^{2}+\left(n+\omega_{F}\right)^{2}}\right\}
$$

where $\omega_{B \pm}=\alpha \pm M_{t}$ and $\omega_{F}=M_{t}$. Here, we rescale all the dimensionful parameters to be dimensionless by using $R$, for example, $M_{t} \rightarrow M_{t} / R$. The numerator of the prefactor, $2 N_{c}$, represents degrees of freedom of a colored complex scalar or a colored Weyl fermion.

To handle this infinite sum, it is convenient to use $W$ and its derivative defined as

$$
W(\omega) \equiv \frac{1}{2} \sum_{n=-\infty}^{\infty} \int \frac{d^{4} k}{(2 \pi)^{4}} \log \frac{k^{2}+(n+\omega)^{2}}{k^{2}+n^{2}}, \quad W^{\prime}(\omega)=\sum_{n=-\infty}^{\infty} \int \frac{d^{4} k}{(2 \pi)^{4}} \frac{(n+\omega)}{k^{2}+(n+\omega)^{2}}
$$

Then we can rewrite the effective potential (3.8),

$$
\begin{aligned}
V_{t} & =\frac{2 N_{c}}{R^{4}}\left[W\left(\omega_{B+}\right)+W\left(\omega_{B-}\right)-2 W\left(\omega_{F}\right)\right] \\
& =\frac{2 N_{c}}{R^{4}}\left[\int_{0}^{\omega_{B+}} d \omega W^{\prime}(\omega)+\int_{0}^{\omega_{B-}} d \omega W^{\prime}(\omega)-2 \int_{0}^{\omega_{F}} d \omega W^{\prime}(\omega)\right] .
\end{aligned}
$$

$W^{\prime}$ is computed with a technique well-known in field theory with finite temperature (see appendix B),

$$
W^{\prime}(\omega)=\frac{-3 i}{2(2 \pi)^{5}}\left[\operatorname{Li}_{4}\left(e^{2 \pi i \omega}\right)-\operatorname{Li}_{4}\left(e^{-2 \pi i \omega}\right)\right],
$$

and hence,

$$
W(\omega)=\frac{-3}{2(2 \pi)^{6}}\left[\operatorname{Li}_{5}\left(e^{2 \pi i \omega}\right)+\operatorname{Li}_{5}\left(e^{-2 \pi i \omega}\right)\right] .
$$

Polylogarithm, $\operatorname{Li}_{s}(z)=\sum_{k=1}^{\infty} \frac{z^{k}}{k^{s}}$, implies the KK tower effect. When $z=1$, it coincides with the Riemann zeta function, $\zeta(s)$. 
The effective potential becomes a simple and finite formula,

$$
\begin{gathered}
V_{t}=\frac{-3 N_{c}}{64 \pi^{6} R^{4}}\left[\operatorname{Li}_{5}\left(e^{2 \pi i \omega_{B+}}\right)+\operatorname{Li}_{5}\left(e^{-2 \pi i \omega_{B+}}\right)+\operatorname{Li}_{5}\left(e^{2 \pi i \omega_{B-}}\right)+\operatorname{Li}_{5}\left(e^{-2 \pi i \omega_{B-}}\right)\right. \\
\left.-2 \operatorname{Li}_{5}\left(e^{2 \pi i \omega_{F}}\right)-2 \operatorname{Li}_{5}\left(e^{-2 \pi i \omega_{F}}\right)\right] .
\end{gathered}
$$

In this calculation, the UV regularization is not needed because the Scherk-Schwarz mechanism, thanks to the non-local supersymmetry breaking, has a noble feature of UV finiteness. Many literatures [29-39] discuss the Scherk-Schwarz mechanism in this context. The UV insensitivity is actually observed in intermediated steps of our computation (appendix B). After the summation of the KK modes, there are two pieces: one has stronger UV divergence which is independent of $\alpha$ and the other has exponentially suppressed UV dependence. Since the former is $\alpha$ independent, the divergence pieces are completely cancelled by combining bosonic and fermionic contributions.

The similar calculation of the effective potential is found in ref. [51] where the Folded Supersymmetry model is used (see also ref. [56] for a mass spectrum with brane terms).

\subsection{Enhancement of Higgs quartic coupling}

The obtained effective potential is now used to get a new Higgs quartic coupling which includes the effect of all the KK modes. First, we put $M_{t}$ back to a dimensionful parameter by extracting $R$ factor and expand the potential with respect to $M_{t}(\ll \alpha / R)$,

$$
\begin{aligned}
V_{t}= & -\frac{3 N_{c}}{32 \pi^{6} R^{4}}\left(\operatorname{Li}_{5}\left(e^{2 \pi i \alpha}\right)+\operatorname{Li}_{5}\left(e^{-2 \pi i \alpha}\right)-2 \zeta(5)\right) \\
& +\frac{3 N_{c}}{16 \pi^{4} R^{2}}\left(\operatorname{Li}_{3}\left(e^{2 \pi i \alpha}\right)+\operatorname{Li}_{3}\left(e^{-2 \pi i \alpha}\right)-2 \zeta(3)\right) M_{t}^{2} \\
& +\frac{N_{c}}{16 \pi^{2}}\left(\frac{25}{6}+\log \left(1-e^{2 \pi i \alpha}\right)\left(1-e^{-2 \pi i \alpha}\right)-2 \log \left(2 \pi M_{t} R\right)\right) M_{t}^{4}+\frac{\mathcal{O}\left(M_{t}^{6} R^{6}\right)}{R^{4}} .
\end{aligned}
$$

The above correction is maximized at $\alpha=1 / 2$. The term of $M_{t}^{4}$ for small $\alpha$ is

$$
\frac{N_{c}}{16 \pi^{2}}\left(\frac{25}{6}+2 \log \frac{\alpha / R}{M_{t}}-\frac{\pi^{2} \alpha^{2}}{3}+\mathcal{O}\left(\alpha^{4}\right)\right) M_{t}^{4} .
$$

The first two terms give the same result of the Higgs quartic coupling radiatively generated by the MSSM (zero mode) particles. The last term, $-\pi^{2} \alpha^{2} / 3$, is from the KK tower but it is negative, which decreases the Higgs mass. However, an important effect of the KK tower comes from $M_{t}^{2}$ term since $M_{t}$ has higher power of $H_{u}$ as shown in eq. (3.5). The potential is expanded with respect to $H_{u}$,

$$
\begin{aligned}
V_{t}= & -\frac{3 N_{c}}{32 \pi^{6} R^{4}}\left(\operatorname{Li}_{5}\left(e^{2 \pi i \alpha}\right)+\operatorname{Li}_{5}\left(e^{-2 \pi i \alpha}\right)-2 \zeta(5)\right) \\
& +\frac{3 y_{t}^{2} N_{c}}{16 \pi^{4} R^{2}}\left(\operatorname{Li}_{3}\left(e^{2 \pi i \alpha}\right)+\operatorname{Li}_{3}\left(e^{-2 \pi i \alpha}\right)-2 \zeta(3)\right) H_{u}^{2} \\
& +\frac{y_{t}^{4} N_{c}}{16 \pi^{2}}\left(\frac{25}{6}+\log \left(1-e^{2 \pi i \alpha}\right)\left(1-e^{-2 \pi i \alpha}\right)-2 \log \left(2 \pi M_{t} R\right)\right. \\
& \left.-2 \operatorname{Li}_{3}\left(e^{2 \pi i \alpha}\right)-2 \operatorname{Li}_{3}\left(e^{-2 \pi i \alpha}\right)+4 \zeta(3)\right) H_{u}^{4}+\frac{\mathcal{O}\left(H_{u}^{6} R^{6}\right)}{R^{4}}
\end{aligned}
$$




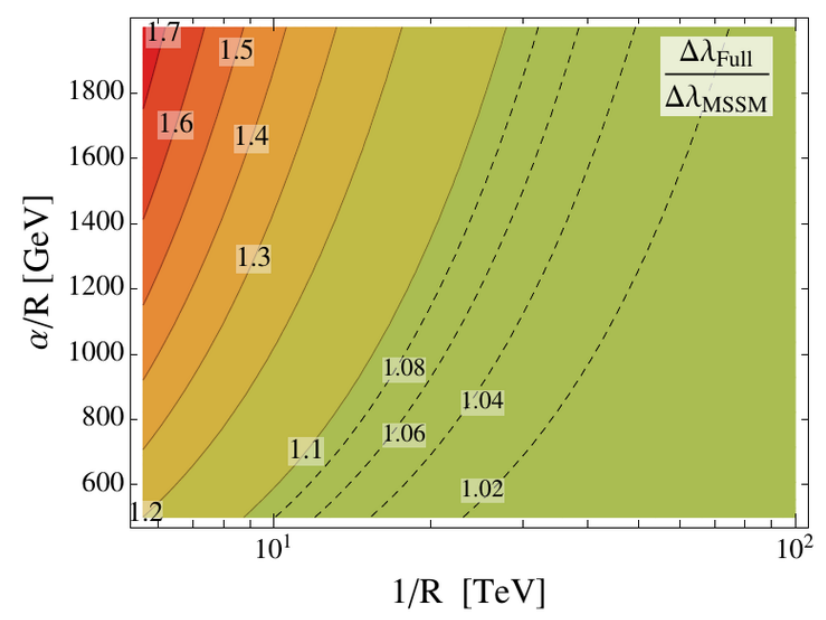

Figure 2. Ratio of 1-loop corrections to $H_{u}^{4}$ coupling. $\Delta \lambda_{\text {Full }}$ is Higgs quartic coupling generated from all the KK modes, while $\Delta \lambda_{\text {MSSM }}$ is that from the zero mode (only the MSSM contribution).

The last line is from $M_{t}^{2}$ term, and it gives a large and positive contribution,

$$
-2 \operatorname{Li}_{3}\left(e^{2 \pi i \alpha}\right)-2 \operatorname{Li}_{3}\left(e^{-2 \pi i \alpha}\right)+4 \zeta(3)=(12-8 \log (2 \pi \alpha)) \pi^{2} \alpha^{2}+\mathcal{O}\left(\alpha^{4}\right) .
$$

For instance, combining with the last term in eq. (3.15), $-\pi^{2} \alpha^{2} / 3$, a total contribution to the Higgs quartic coupling from the KK tower is proportional to $(-1 / 3+12-$ $8 \log (2 \pi \alpha)) \pi^{2} \alpha^{2}=3.3 \times 10^{-2}-3.9$ for parameter of our interest, $\alpha=10^{-2}-0.2$, which can be compared to the MSSM contribution $\approx 25 / 6$. To see the impact of this new effect, figure 2 shows a comparison between $\Delta \lambda_{\text {Full }}$ defined as the Higgs quartic coupling in eq. (3.16) and the Higgs quartic coupling in the MSSM, $\Delta \lambda_{\mathrm{MSSM}}$, defined by

$$
\Delta \lambda_{\mathrm{MSSM}} H_{u}^{4} \equiv \frac{y_{t}^{4} N_{c}}{16 \pi^{2}}\left(\frac{25}{6}+2 \log \frac{\alpha / R}{M_{t}}\right) H_{u}^{4}
$$

where $M_{t} \ll \alpha / R$ is assumed. It is shown in figure 2 that even for a $10 \mathrm{TeV}$ scale of the extra dimension, the effect of the KK modes enhances $10 \sim 50 \%$ radiative correction to the quartic coupling. This is surprising because, if the MSSM has mass scale of $\alpha / R \sim \mathrm{TeV}$, modification from the KK modes is naively expected to be $(\alpha / R)^{2} / R^{-2} \sim 1 \%$ rather than $\mathcal{O}(10 \%)$. Therefore this new contribution will give a big change to the calculation of the lightest Higgs mass.

Note that terms with higher power of $H_{u}$, such as $H_{u}^{6}$, are not important for the Higgs mass when we consider parameter space of $v \ll R^{-1}$ hence $H_{u} R$ expansion is valid. We explicitly show this in appendix C.

\section{Higgs mass}

We found the Higgs quartic coupling enhanced by the full KK tower. Then we need electroweak symmetry breaking parameters, such as $\tan \beta \equiv\left\langle H_{u}\right\rangle /\left\langle H_{d}\right\rangle$, to evaluate the lightest Higgs mass, $m_{h}$. In the following section 4.1 , we obtain $\mu$ and $\tan \beta$ by solving 


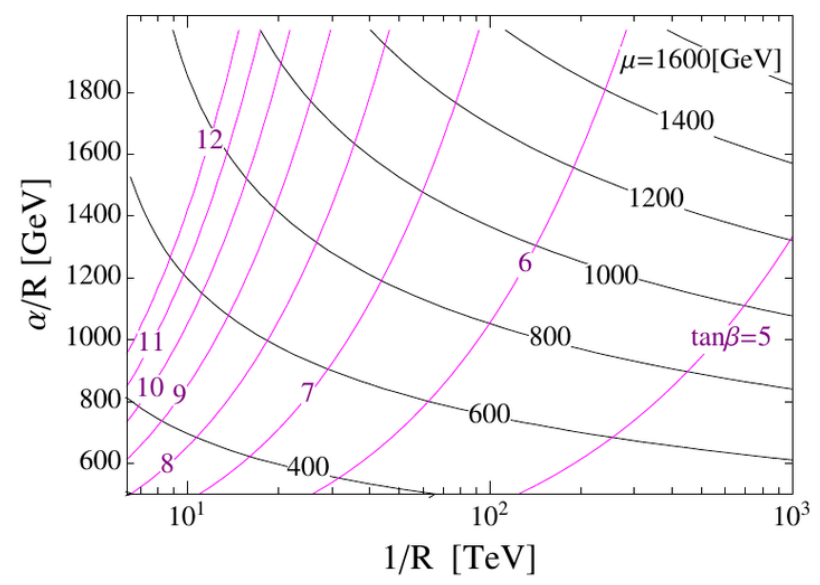

Figure 3. $\mu$ and $\tan \beta$ are determined by requiring the successful electroweak symmetry breaking. Black lines show $\mu$ in $\mathrm{GeV}$ and purple lines show $\tan \beta$.

electroweak symmetry breaking conditions. In section 4.2, we improve 1-loop calculation from the top sector by RGE to include next-to-leading logarithm order and also take into account the leading correction of electroweak gauge couplings. Finally, in section 4.3, we present results of the Higgs mass and fine-tuning.

\subsection{Electroweak symmetry breaking}

Despite the constrained structure of the model, successful electroweak symmetry breaking can be achieved. Since the scale of the extra dimension is well above the supersymmetry breaking scale, we match the theory onto the MSSM with a matching (renomalization) scale,

$$
Q_{\mathrm{RG}}=\frac{1}{2 \pi R} .
$$

Higgs soft terms are absent at tree level but are generated radiatively. We calculate those corrections including all the KK modes which are finite thanks to the Scherk-Schwarz mechanism, and subtract the MSSM parts which are regularized with the $\overline{\mathrm{DR}}$ scheme from them. As a result, threshold corrections to the Higgs soft terms at the matching scale are

$$
\begin{aligned}
m_{H_{u}}^{2} & =\left(-\frac{3 y_{t}^{2}}{\pi^{2}}+\frac{3\left(g_{2}^{2}+g_{1}^{2} / 5\right)}{8 \pi^{2}}\right)\left(\frac{\alpha}{R}\right)^{2}, \\
m_{H_{d}}^{2} & =\frac{3\left(g_{2}^{2}+g_{1}^{2} / 5\right)}{8 \pi^{2}}\left(\frac{\alpha}{R}\right)^{2}, \\
b & =\left(\frac{3 y_{t}^{2}}{4 \pi^{2}}-\frac{3\left(g_{2}^{2}+g_{1}^{2} / 5\right)}{16 \pi^{2}}\right) \mu \frac{\alpha}{R} .
\end{aligned}
$$

The detail of these results is given in appendix D. We solve electroweak symmetry breaking conditions using SOFTSUSY 3.4 [57]. Among three free parameters of the model, one of them is determined by the Higgs VEV. In figure $3, \mu$ as well as $\tan \beta$ are shown in $\alpha / R$ and $1 / R$ parameter space. 
We find the Higgsino mass scale, $\mu$, has to be close to gaugino, squark, and slepton mass scale, $\alpha / R$, which leads to a compressed spectrum which ameliorates LHC bounds. The spectrum is more compressed as $R^{-1}$ gets large because $\mu$ grows to compensate $-\left|m_{H_{u}}^{2}\right|$ which becomes larger due to a long RGE running. Since $\tan \beta$ is found to be as low as $\tan \beta \lesssim 10$ in the parameter space, the tree level Higgs mass, $\left|m_{Z} \cos (2 \beta)\right|$, is rather low and hence a large radiative correction is needed to realize the observed value $m_{h} \approx 125 \mathrm{GeV}$.

\subsection{Higher order corrections}

It is known that the corrections at $\mathcal{O}\left(y_{t}^{4} g_{s}^{2}, y_{t}^{6}\right)$ are significant and this is mainly due to the scale of the top mass. Here we adopt a RG-improved method [58] (see also refs. [59-61]) based on our 1-loop calculation. The top mass (top Yukawa and $H_{u}$ VEV) runs to an intermediate scale of top quark and stop by

$$
y_{t} \rightarrow y_{t}\left(M_{t}\right)\left(1+\beta_{t} \log \frac{Q}{M_{t}}\right), \quad v_{u} \rightarrow v_{u}\left(M_{t}\right)\left(1+\gamma_{v_{u}} \log \frac{Q}{M_{t}}\right),
$$

where

$$
Q=c_{t} \sqrt{M_{t}\left(M_{t}^{2}+\alpha^{2} / R^{2}\right)^{1 / 2}} .
$$

The beta function and anomalous dimension are those for the two-Higgs Doublet Model,

$$
\beta_{t}=\frac{9 y_{t}^{2}}{32 \pi^{2}}-\frac{g_{s}^{2}}{2 \pi^{2}}, \quad \gamma_{v_{u}}=-\frac{3 y_{t}^{2}}{16 \pi^{2}} .
$$

We choose $c_{t}=2.1$ so that this RG-improved calculation for the MSSM Higgs mass reproduces the 2-loop result calculated by FeynHiggs [62] to a good accuracy. This top mass is used for the formulae for the Higgs quartic coupling in eqs. (3.16), (3.18). Regarding the KK mode contribution, say $\Delta \lambda_{\mathrm{KK}} H_{u}^{4} \equiv\left(\Delta \lambda_{\text {Full }}-\Delta \lambda_{\mathrm{MSSM}}\right) H_{u}^{4}$, a more appropriate treatment is that $\Delta \lambda_{\mathrm{KK}} H_{u}^{4}$ is given at the KK scale of $1 / R$ and runs down to the scale $\alpha / R$. However, since such an effect is subdominant and requires to consider mixing with other operators, it is beyond the scope of this paper.

In order to further improve our computation to the Higgs mass, we include $\mathcal{O}\left(y_{t}^{2} g_{1}^{2}, y_{t}^{2} g_{2}^{2}\right)$ correction. Here, we focus on the MSSM contribution and the corresponding correction to the Higgs potential is

$$
V_{y_{t}^{2} g^{2}}=-\frac{g_{1}^{2}+g_{2}^{2}}{4} \frac{3 y_{t}^{2}}{8 \pi^{2}} \log \frac{m_{\tilde{t}}^{2}+M_{t}^{2}}{M_{t}^{2}}\left(\left|H_{u}\right|^{4}-\frac{1}{2}\left|H_{u}\right|^{2}\left|H_{d}\right|^{2}\right)
$$

where we neglect the mixing between $\tilde{t}_{R}$ and $\tilde{t}_{L}$. The top Yukawa here is also given by eq. (4.5).

\subsection{Enhanced Higgs mass}

Based on the RG-improved method and electroweak parameters, we calculate the Higgs mass. In figure 4, we show the Higgs mass calculated at the MSSM level and that calculated with the full KK tower. Even for heavy KK modes of $R^{-1} \sim 10 \mathrm{TeV}$, a line of $m_{h}=$ $125 \mathrm{GeV}$ based on the MSSM is underestimated because once the full KK tower is included, the Higgs mass is significantly enhanced and then the line of $m_{h}=125 \mathrm{GeV}$ based on the MSSM actually corresponds to $m_{h}=130-140 \mathrm{GeV}$. The true line of $m_{h}=125 \mathrm{GeV}$ is realized in a lower supersymmetry breaking scale. The line becomes a band if we consider 


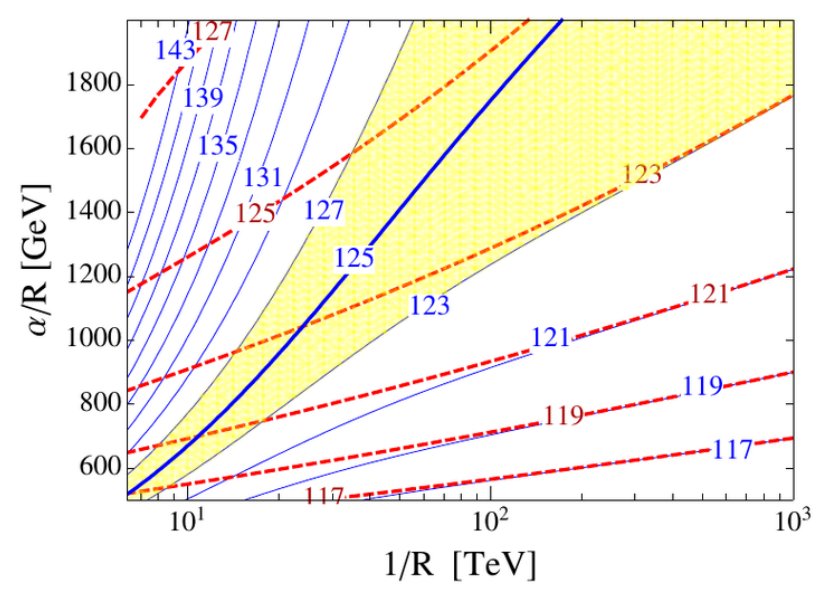

Figure 4. The lightest Higgs mass in an unit of GeV. Each blue solid line is Higgs mass calculated with the full KK tower, and each red dashed line corresponds to Higgs mass based on the MSSM calculation. The MSSM calculation always underestimates the Higgs mass. The yellow band is a region of $m_{h}=125 \pm 2 \mathrm{GeV}$.

an uncertainty of our prediction, and we take conservatively $2 \mathrm{GeV}$ as an uncertainty of the Higgs mass which corresponds to a band in figure 4. As in figure 8 of the Higgs quartic coupling, the effect of KK modes eventually disappears when $R^{-1}$ goes beyond $100 \mathrm{TeV}(\alpha \lesssim 0.01)$.

Improvement of Higgs mass calculation is very important in the Compact Supersymmetry model because it has only three free parameters, $R, \alpha / R$, and $\mu$, one of which is determined by the Higgs VEV, and furthermore the observed Higgs mass constraints one more parameter leading to just a line (a band with an error) in the parameter space. It is shown that the MSSM calculation points to wrong region in the parameter space. The improved calculation tells that supersymmetry breaking scale that explains the Higgs mass is lowered, which motivates $\mathrm{TeV}$ supersymmetry signature with a compressed spectrum. Also, testability of the model at the LHC increases.

Fine-tuning is also investigated. For the successful electroweak symmetry breaking, $\mu$ has to be almost as big as the supersymmetry breaking scale, $\alpha / R$, which leads to a tree level tuning of the weak scale due to $\mu \gg v, m_{Z}$. Since $\mu$ is the dominant source of fine-tuning to realize the correct weak scale as pointed out in ref. [19], we adopt a simple fine-tuning measure, $\Delta_{\mu}^{-1}$, varying only $\mu$,

$$
\Delta_{\mu}^{-1}=\left|\frac{\partial \log v^{2}}{\partial \log \mu}\right|^{-1} \approx \frac{m_{h}^{2}}{4 \mu^{2}} .
$$

The last approximation is valid when heavy Higgs states are decoupled [48]. In this case, the Higgs sector corresponds to SM-like one-Higgs doublet model, and we can derive

$$
\frac{\partial v^{2}}{\partial \mu^{2}}=\frac{v^{2}}{m_{h}^{2}} \frac{\partial m_{h}^{2}}{\partial \mu^{2}}=\frac{-2 v^{2}}{m_{h}^{2}} .
$$

Using this approximation, in figure 5, we show fine-tuning of the Compact Supersymmetry model is percent level. 


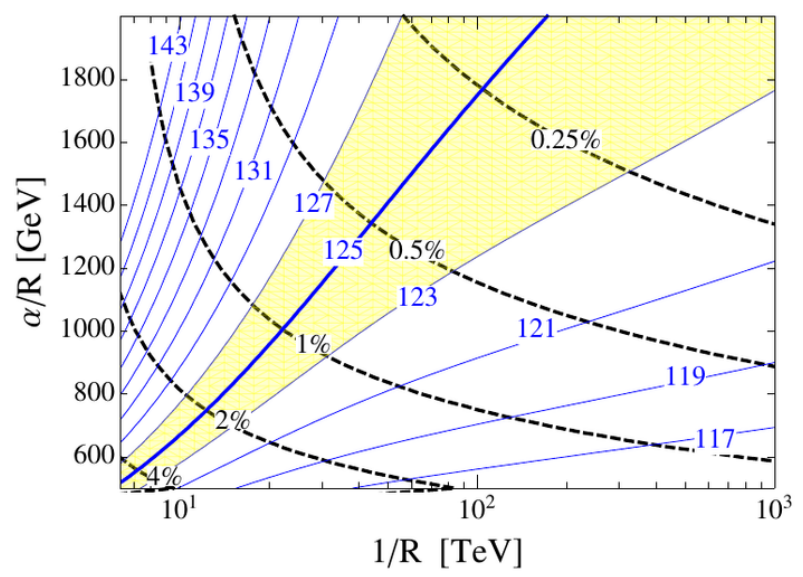

Figure 5. Fine-tuning $\Delta_{\mu}^{-1} \approx m_{h}^{2} /\left(4 \mu^{2}\right)$ is plotted as black dashed lines. Blue solid lines correspond to the lightest Higgs mass in a unit of GeV calculated with the full KK tower, and the yellow band is a region of $m_{h}=125 \pm 2 \mathrm{GeV}$.

\section{$5 \quad$ Experimental bounds}

In this section, we discuss how experimental results bound on the parameter space. The first constraint we consider is from the electroweak precision test. Since this model has brane-localized Higgs, the Higgs VEV mixes zero mode and non-zero modes of electroweak gauge bosons at tree level. Refs. [44, 45] study such bounds and lead to a limit on a size of the extra dimension, $R^{-1} \gtrsim 5 \mathrm{TeV}$. It does not constrain interesting parameter space which can explain the Higgs mass and the current LHC results.

Secondly, the ATLAS and CMS experiments search for supersymmetric particles and derive bounds in many channels. Here we study a representative analysis of multijet $+\mathbb{E}_{T}$. Since the Compact Supersymmetry model has a compressed spectrum whose mass difference between the LSP and gluino/squark is typically $300 \mathrm{GeV}$, the bound is weaker than those for the CMSSM and simplified models $\left(m_{\tilde{g}} \approx m_{\tilde{q}}\right)$ [17]. For the spectrum calculation, as discussed in section 4.1, we match the theory onto the MSSM at a scale of $1 /(2 \pi R)$ and consider RGE running down to the supersymmetry breaking scale, $\alpha / R$. We generate signal events by PYTHIA 6.4 [63], and use PGS 4 [64] for the detector simulation and NLL-fast [65-70] for estimation of the production cross section including next-to-leading order QCD corrections and the resummation at next-to-leading-logarithmic accuracy. We compare the obtained event numbers with ATLAS searches using multijet $+\mathbb{E}_{T}$ without lepton with $\mathcal{L}=20.3 \mathrm{fb}^{-1}$ at $\sqrt{s}=8 \mathrm{TeV}$ [17], and the result is shown as a lower shaded region in figure 6 . We find the exclusion bound is extended up to $m_{\tilde{g}} \simeq 1 \mathrm{TeV}$, and for a region at $R^{-1} \sim 10 \mathrm{TeV}$ the bound is stronger as $m_{\tilde{g}} \gtrsim 1.3 \mathrm{TeV}$ because the spectrum is less compressed in this region. In contrast, the CMSSM and simplified model are more constrained as the bound is $m_{\tilde{g}} \gtrsim 1.7 \mathrm{TeV}$.

Finally, the LSP can be a dark matter candidate. As long as the LSP is stable, its relic abundance should be lower than the observed dark matter relic abundance. We calculate thermal relic abundance of the LSP using Micro0MEGAs [71, 72], and put a 95\% C.L. upper 


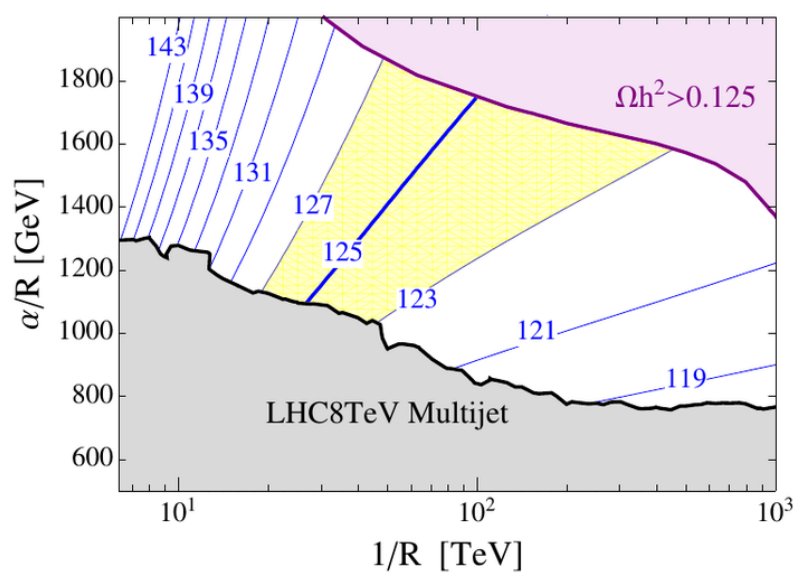

Figure 6. The upper (purple) shaded region is excluded by the thermal relic abundance of the LSP larger than the observed dark matter relic abundance, $\Omega h^{2}>0.125$. The lower (gray) shaded region is excluded by one of the ATLAS results which are based on multijet $+\mathbb{E}_{T}$. Blue solid lines correspond to the lightest Higgs mass in a unit of $\mathrm{GeV}$ calculated with all the KK tower, and the yellow band is a region of $m_{h}=125 \pm 2 \mathrm{GeV}$.

bound on the relic abundance, $\Omega h^{2}<0.125$, obtained by Planck collaboration [73]. The LSP in this model is dominated by the Higgsino component because of $\mu<\alpha / R$, and then the thermal relic abundance of the LSP is smaller than the observed abundance unless it is heavy to decouple from thermal bath earlier. The excluded region is shown as an upper shaded region in figure 6 . It is very interesting because this tells that the model can be tested, that is, along the Higgs mass band there is upper bound in the $\mathrm{TeV}$ range by the dark matter relic abundance and the LHC bound from the bottom will be improved at upcoming LHC run at $\sqrt{s}=13$ and $14 \mathrm{TeV}$.

Regarding the future search for models with a compressed spectrum such as the Compact Supersymmetry model, since the signal is weaker, it is important to improve the sensitivity. One possibility is to utilize $M_{T 2}$ [74] which can systematically separate signal and background, and its validity is demonstrated in ref. [75] that $M_{T 2}$ significantly improves discovery potential of the Minimal Universal Extra Dimension [76] which has a compressed spectrum. Also, other useful techniques [77-83] are developed to improve the sensitivity to models with compressed spectra at the LHC. Using these techniques and all channels of experiments, we believe the whole parameter space of the Compact Supersymmetry model compatible with the observed Higgs mass and the dark matter relic abundance $(\alpha / R \lesssim 1.8 \mathrm{TeV})$ is explicitly testable at the LHC.

\section{Conclusions}

We studied an impact of the KK tower to the lightest Higgs mass in the Compact Supersymmetry model. We computed the effective potential of all the KK modes (with Higgs dependent mass eigenvalues), and find the enhancement of the Higgs quartic coupling is unexpectedly large. The effect of the KK modes enhances the radiative contribution to the 
Higgs quartic coupling by from 10 to $50 \%$ even for heavy KK modes of $\mathcal{O}(10) \mathrm{TeV}$, and the effect remains non-negligible until $\mathcal{O}(100) \mathrm{TeV}$. This is mainly because the top quark wave function is pushed out from the brane, which makes the top mass depend on higher powers in the Higgs field. Correspondingly, the Higgs mass is raised by from 5 to $15 \mathrm{GeV}$, and hence the Higgs mass of $125 \mathrm{GeV}$ is realized in a lower supersymmetry breaking scale of $\alpha / R$. The better knowledge of the Higgs mass together with the Higgs VEV essentially leaves only one free parameter of the model. Furthermore the parameter space is bounded, with respect to $\alpha / R$, from the bottom by the LHC searches at $\alpha / R \simeq 1 \mathrm{TeV}$ and from the top by the the dark matter relic abundance at $\alpha / R \simeq 1.8 \mathrm{TeV}$. Although the compressed spectrum weakens LHC bounds, since the LHC run II can investigate higher mass scale by the higher energy, the whole parameter space of the model will be explicitly tested.

\section{Acknowledgments}

We thank Lorenzo di Pietro, Hou Keong Lou, Xiaochuan Lu, Yasunori Nomura, and Ryosuke Sato for useful discussions. This work was supported by JSPS KAKENHI Grantin-Aid for Scientific Research (B) (No. 15H03669 [RK]) and (C) (No. 26400241 [HM]), Grant-in-Aid for JSPS Fellows (No. 14J00179 [KT]), MEXT KAKENHI Grant-in-Aid for Scientific Research on Innovative Areas (No. 25105011 [RK], No. 15H05887 [HM]), and by WPI, MEXT, Japan. HM also was supported in part by the U.S. DOE under Contract DE-AC03-76SF00098, in part by the NSF under grant PHY-1316783.

\section{A Mass spectrum}

\section{A.1 Equations of motion}

In order to obtain Higgs-dependent mass eigenvalues, we solve 5D equations of motion and find wave functions. Equations of motion for squark are

$$
\begin{aligned}
\left(-\partial^{2}+\partial_{5}^{2}\right) \tilde{Q}-y_{U 5}^{2} H_{u}^{2} \delta(y) \delta(0) \tilde{Q}+y_{U 5} H_{u} \delta(y) \partial_{5} \tilde{U}^{c} & =0 \\
\left(-\partial^{2}+\partial_{5}^{2}\right) \tilde{U}^{c}-y_{U 5} H_{u} \partial_{5}[\delta(y) \tilde{Q}] & =0 \\
\left(-\partial^{2}+\partial_{5}^{2}\right) \tilde{U}^{*}-y_{U 5}^{2} H_{u}^{2} \delta(y) \delta(0) \tilde{U}^{*}+y_{U 5} H_{u} \delta(y) \partial_{5} \tilde{Q}^{c *} & =0 \\
\left(-\partial^{2}+\partial_{5}^{2}\right) \tilde{Q}^{c *}-y_{U 5} H_{u} \partial_{5}\left[\delta(y) \tilde{U}^{*}\right] & =0 .
\end{aligned}
$$

Since we are only interested in top quark, $\tilde{Q}^{(c)}$ represents $5 \mathrm{D}$ top squark field in the $\mathrm{SU}(2)_{L}$ doublet and $\tilde{U}^{(c)}$ also represents 5D top squark field, and therefore Yukawa coupling here corresponds to top Yukawa, $y_{U 5} \rightarrow y_{t 5}$. All the parameters are taken to be real for simplicity. In the following, we perform $4 \mathrm{D}$ Fourier transformation and consider on-shell, that is, $-\partial^{2} \rightarrow p^{2}=M^{2}$.

Equations of motion for quark are

$$
\begin{aligned}
& \left(i \not \partial+\gamma_{5} \partial_{5}\right) \Psi_{Q}+\delta(y) y_{U 5} P_{R} \Psi_{U}^{C} H_{u}=0, \\
& \left(i \not \partial+\gamma_{5} \partial_{5}\right) \Psi_{U}+\delta(y) y_{U 5} P_{R} \Psi_{Q}^{C} H_{u}=0,
\end{aligned}
$$

where the superscript $C$ denotes charge conjugation of the fermion. As in the squark case, we focus on top quark. 


\section{A.2 Solution for quark}

We can separate quark wave functions to $4 \mathrm{D}$ parts and extra dimensional parts,

$$
\begin{aligned}
& \Psi_{Q}(x, y)=\left(\begin{array}{c}
Q(x, y) \\
\bar{Q}^{c}(x, y)
\end{array}\right)=\psi_{Q-}(x) f_{Q-}(y)+\psi_{Q+}(x) f_{Q+}(y) \\
& \Psi_{U}(x, y)=\left(\begin{array}{c}
U(x, y) \\
\bar{U}^{c}(x, y)
\end{array}\right)=\psi_{U-}(x) f_{U-}(y)+\psi_{U+}(x) f_{U+}(y) .
\end{aligned}
$$

$\psi_{ \pm}$is four component spinor which has a chirality, $\gamma_{5} \psi_{ \pm}= \pm \psi_{ \pm}$. Under the reflection, $\mathcal{Z}: y \rightarrow-y, f_{Q-}(y)$ and $f_{Q+}(y)$ should have the same transformations of $Q(x, y)$ and $\bar{Q}^{c}(x, y)$, respectively, as

$$
f_{Q-}(-y)=+f_{Q-}(y), \quad f_{Q+}(-y)=-f_{Q+}(y) .
$$

This condition is same for $U$ quark fields.

Now we investigate the bulk Lagrangian omitting arguments for simplicity,

$$
\begin{aligned}
\bar{\Psi}_{Q}\left(i \not \partial+\gamma_{5} \partial_{5}\right) \Psi_{Q}= & \left(\bar{\psi}_{Q-} f_{Q-}^{*}+\bar{\psi}_{Q+} f_{Q+}^{*}\right)\left(i \not \partial+\gamma_{5} \partial_{5}\right)\left(\psi_{Q-} f_{Q-}+\psi_{Q+} f_{Q+}\right) \\
= & \left(\bar{\psi}_{Q-} i \not \partial \psi_{Q-}\right)\left(f_{Q-}^{*} f_{Q-}\right)+\left(\bar{\psi}_{Q+} i \not \partial \psi_{Q+}\right)\left(f_{Q+}^{*} f_{Q+}\right) \\
& +\left(\bar{\psi}_{Q-} \gamma_{5} \psi_{Q+}\right)\left(f_{Q-}^{*} \partial_{5} f_{Q+}\right)+\left(\bar{\psi}_{Q+} \gamma_{5} \psi_{Q-}\right)\left(f_{Q+}^{*} \partial_{5} f_{Q-}\right) .
\end{aligned}
$$

EOMs in the bulk lead to

$$
\begin{aligned}
& i \not \partial \psi_{Q-}\left(f_{Q-}^{*} f_{Q-}\right)=-\psi_{Q+}\left(f_{Q-}^{*} \partial_{5} f_{Q+}\right), \\
& i \not \partial \psi_{Q+}\left(f_{Q+}^{*} f_{Q+}\right)=\psi_{Q-}\left(f_{Q+}^{*} \partial_{5} f_{Q-}\right) .
\end{aligned}
$$

The last equality is obtained by integration by parts. Separation of variables is used,

$$
\begin{gathered}
\frac{i \not \partial \psi_{Q-}(x)}{\psi_{Q+}(x)}=M=-\frac{\partial_{5} f_{Q+}(y)}{f_{Q-}(y)}, \\
\frac{i \not \psi_{Q+}(x)}{\psi_{Q-}(x)}=M^{\prime}=\frac{\partial_{5} f_{Q-}(y)}{f_{Q+}(y)} .
\end{gathered}
$$

$M$ and $M^{\prime}$ constant and interpreted as 4D quark mass, and $M=M^{\prime}$ is necessary because $\psi_{Q+}$ and $\psi_{Q-}$ behave as a single Dirac fermion. Then, a differential equation for $f_{Q-}(y)$ is

$$
\partial_{5}^{2} f_{Q-}(y)=\partial_{5}\left(M f_{Q+}(y)\right)=-M^{2} f_{Q-}(y),
$$

and a general solution of $f_{Q-}$ in the bulk is

$$
f_{Q-}(y)=A \cos (M y)+B \operatorname{sign}(y) \sin (M y) .
$$

A solution of $f_{Q+}$ is given by $f_{Q+}(y)=\partial_{5} f_{Q-}(y) / M$,

$$
f_{Q+}(y)=-A \sin (M y)+B \operatorname{sign}(y) \cos (M y) .
$$


For $U$ quark, we have similar conditions, ${ }^{2}$

$$
M=\frac{i \not \psi_{U-}(x)}{\psi_{U+}(x)}=-\frac{\partial_{5} f_{U+}(y)}{f_{U-}(y)}=\frac{i \not \partial \psi_{U+}(x)}{\psi_{U-}(x)}=\frac{\partial_{5} f_{U-}(y)}{f_{U+}(y)}
$$

and here are solutions,

$$
\begin{aligned}
& f_{U-}(y)=C \cos (M y)+D \operatorname{sign}(y) \sin (M y), \\
& f_{U+}(y)=-C \sin (M y)+D \operatorname{sign}(y) \cos (M y) .
\end{aligned}
$$

For the case of quark, we can define another reflection, $\mathcal{Z}^{\prime}: y+\pi R \rightarrow-y+\pi R$, that is a product of $\mathcal{T}$ and $\mathcal{Z}\left(\mathcal{Z}^{\prime}=\mathcal{Z} \mathcal{T}\right)$. For fermions of $\Phi^{c}$ which are odd under $\mathcal{Z}$, they are also odd under $\mathcal{Z}^{\prime}$ because of $\mathcal{T}=1$. In particular, $Q^{c}(\pi R)=U^{c}(\pi R)=0$ gives

$$
\begin{aligned}
& A \sin (M \pi R)=B \cos (M \pi R), \\
& C \sin (M \pi R)=D \cos (M \pi R) .
\end{aligned}
$$

We determine the coefficients by integrating EOM of eq. (A.5) around $y=0$ with an infinitesimal interval of $\epsilon$,

$$
0=\int_{-\epsilon}^{\epsilon} d y\left\{\left(i \not \partial+\gamma_{5} \partial_{5}\right) \Psi_{Q}(y)+\delta(y) y_{U 5} P_{R} \Psi_{U}^{C}(y) H_{u}\right\}
$$

The $4 \mathrm{D}$ kinetic term does not give a constraint because it automatically vanishes,

$$
\int_{-\epsilon}^{\epsilon} d y i \not \partial \Psi_{Q}(y)=\int_{-\epsilon}^{\epsilon} d y\left\{M \psi_{Q+} f_{Q-}(y)+M \psi_{Q-} f_{Q+}(y)\right\}=0
$$

We focus on the right-handed component of eq. (A.23),

$$
\begin{aligned}
0 & =\int_{-\epsilon}^{\epsilon} d y\left\{\psi_{Q+} \partial_{5} f_{Q+}(y)+\delta(y) y_{U 5} \psi_{U-}^{C} f_{U-}^{*}(y) H_{u}\right\} \\
& =\psi_{Q+} f_{Q+}(\epsilon)-\psi_{Q+} f_{Q+}(-\epsilon)+y_{U 5} \psi_{U-}^{C} f_{U-}^{*}(0) H_{u} \\
& =2 \psi_{Q+} B+y_{U 5} H_{u} \psi_{U-}^{C} C^{*}
\end{aligned}
$$

The other fermion EOM of eq. (A.6) gives a similar condition,

$$
2 \psi_{U+} D+y_{U 5} H_{u} \psi_{Q-}^{C} A^{*}=0 .
$$

This is modified using eq. (A.18),

$$
2 i \not \partial \psi_{U+} D+y_{U_{5}} H_{u} i \not \partial \psi_{Q-}^{C} A^{*}=2 M \psi_{U-} D+y_{U 5} H_{u} M \psi_{Q+}^{C} A^{*}=0 .
$$

Finally, eqs. (A.21), (A.22), (A.25), (A.27) lead to

$$
\begin{aligned}
\psi_{Q+} A \sin (M \pi R) & =-\frac{y_{U 5} H_{u}}{2} \psi_{U-}^{C} C^{*} \cos (M \pi R) \\
& =\left(\frac{y_{U 5} H_{u}}{2}\right)^{2} \psi_{Q+} A \frac{\cos ^{2}(M \pi R)}{\sin (M \pi R)} .
\end{aligned}
$$

\footnotetext{
${ }^{2}$ The reason that the constant $M$ here is common with one for $Q$ is because we later obtain conditions that $\psi_{Q}$ and $\psi_{U}^{c}$ are related in eqs. (A.25), (A.26). In other words, they are mixed by Higgs VEV.
} 
Hence, a consistency condition of quark mass is

$$
\tan ^{2}(M \pi R)=\left(\frac{y_{U 5} H_{u}}{2}\right)^{2}
$$

The lowest mass eigenvalue gives top quark mass which is not simply proportional to Higgs,

$$
M_{t}\left(H_{u}\right) \equiv \frac{1}{\pi R} \arctan \left(\frac{y_{t 5} H_{u}}{2}\right),
$$

and general mass eigenvalues of top quark are

$$
M=\frac{n}{R} \pm M_{t}\left(H_{u}\right) \quad(n: \text { integer }) .
$$

These eigenvalues are used to calculate the effective potential.

We give the KK expansion for top quark for completeness,

$$
\begin{aligned}
\Psi_{Q}= & \sum_{n=-\infty}^{\infty} N_{f} t_{L}^{(n)}(x)\left[\cos \left(\frac{n}{R}+M_{t}\right) y+\tan \left(M_{t} \pi R\right) \operatorname{sign}(y) \sin \left(\frac{n}{R}+M_{t}\right) y\right] \\
& +\sum_{n=-\infty}^{\infty} N_{f} t_{R}^{(n)}(x)\left[-\sin \left(\frac{n}{R}+M_{t}\right) y+\tan \left(M_{t} \pi R\right) \operatorname{sign}(y) \cos \left(\frac{n}{R}+M_{t}\right) y\right], \\
\Psi_{U}^{C}= & \sum_{n=-\infty}^{\infty} N_{f} t_{R}^{(n)}(x)\left[\cos \left(\frac{n}{R}+M_{t}\right) y+\tan \left(M_{t} \pi R\right) \operatorname{sign}(y) \sin \left(\frac{n}{R}+M_{t}\right) y\right] \\
& +\sum_{n=-\infty}^{\infty} N_{f} t_{L}^{(n)}(x)\left[-\sin \left(\frac{n}{R}+M_{t}\right) y+\tan \left(M_{t} \pi R\right) \operatorname{sign}(y) \cos \left(\frac{n}{R}+M_{t}\right) y\right],
\end{aligned}
$$

where $N_{f}=\frac{\cos \left(M_{t} \pi R\right)}{(2 \pi)^{1 / 2}}$ is a normalization factor.

\section{A.3 Solutions for squarks}

Similarly to the quark case, we solve Klein-Gordon equations for squarks in the bulk respecting properties of $\mathcal{Z}$ reflection,

$$
\begin{aligned}
\tilde{Q}(y) & =A_{1} \cos (M y)+B_{1} \operatorname{sign}(y) \sin (M y), \\
\tilde{U}^{c}(y) & =C_{1} \operatorname{sign}(y) \cos (M y)+D_{1} \sin (M y), \\
\tilde{U}^{*}(y) & =A_{2} \cos (M y)+B_{2} \operatorname{sign}(y) \sin (M y), \\
\tilde{Q}^{c *}(y) & =C_{2} \operatorname{sign}(y) \cos (M y)+D_{2} \sin (M y) .
\end{aligned}
$$

These profiles of eqs. (A.34)-(A.37) are valid for an interval of $-2 \pi R \leq y \leq 2 \pi R$. In principle $\delta(y)$ terms are allowed for $\tilde{Q}$ and $\tilde{U}$, but we neglect those because we can easily show these should terms vanish by EOMs. Discontinuity at $y=0$ is necessary to take into account Higgs effect localized on the brane. For the translation, squarks have twisted boundary conditions,

$$
\begin{aligned}
& \left(\begin{array}{c}
\tilde{Q}(y+2 \pi R) \\
\tilde{Q}^{c *}(y+2 \pi R)
\end{array}\right)=\left(\begin{array}{cc}
\cos (2 \pi \alpha) & \sin (2 \pi \alpha) \\
-\sin (2 \pi \alpha) & \cos (2 \pi \alpha)
\end{array}\right)\left(\begin{array}{c}
\tilde{Q}(y) \\
\tilde{Q}^{c *}(y)
\end{array}\right) \\
& \left(\begin{array}{c}
\tilde{U}(y+2 \pi R) \\
\tilde{U}^{c *}(y+2 \pi R)
\end{array}\right)=\left(\begin{array}{cc}
\cos (2 \pi \alpha) & \sin (2 \pi \alpha) \\
-\sin (2 \pi \alpha) & \cos (2 \pi \alpha)
\end{array}\right)\left(\begin{array}{c}
\tilde{U}(y) \\
\tilde{U}^{c *}(y)
\end{array}\right) .
\end{aligned}
$$


We determine the coefficients of the general solutions by EOMs and the above twisted boundary conditions. Here, only two EOMs of eqs. (A.1), (A.3) are relevant. We consider integral of eq. (A.1) around $y=0$,

$$
\begin{aligned}
0 & =\int_{-\epsilon}^{\epsilon} d y\left\{\left(M^{2}+\partial_{5}^{2}\right) \tilde{Q}(y)-y_{U 5}^{2} H_{u}^{2} \delta(y) \delta(0) \tilde{Q}(y)+y_{U 5} H_{u} \delta(y) \partial_{5} \tilde{U}^{c}(y)\right\} \\
& =\int_{-\epsilon}^{\epsilon} d y\left\{M^{2} \tilde{Q}(y)\right\}+\partial_{5} \tilde{Q}(\epsilon)-\partial_{5} \tilde{Q}(-\epsilon)-y_{U 5}^{2} H_{u}^{2} \delta(0) \tilde{Q}(0)+y_{U 5} H_{u} \partial_{5} \tilde{U}^{c}(0) \\
& =2 M B_{1}-y_{U 5}^{2} H_{u}^{2} \delta(0) A_{1}+y_{U 5} H_{u}\left(2 \delta(0) C_{1}+M D_{1}\right) .
\end{aligned}
$$

Therefore comparing coefficients leads to two conditions,

$$
C_{1}=\frac{y_{U 5} H_{u}}{2} A_{1}, \quad B_{1}=-\frac{y_{U 5} H_{u}}{2} D_{1} .
$$

Similarly, eq. (A.3) gives

$$
C_{2}=\frac{y_{U 5} H_{u}}{2} A_{2}, \quad B_{2}=-\frac{y_{U 5} H_{u}}{2} D_{2} .
$$

Next we apply boundary conditions of the translation. The condition for $\tilde{Q}$ of eq. (A.38) leads to

$$
\begin{aligned}
\left(\begin{array}{c}
\tilde{Q}(2 \pi R-\epsilon) \\
\tilde{Q}^{c *}(2 \pi R-\epsilon)
\end{array}\right) & =\left(\begin{array}{cc}
\cos (2 \pi \alpha) & \sin (2 \pi \alpha) \\
-\sin (2 \pi \alpha) & \cos (2 \pi \alpha)
\end{array}\right)\left(\begin{array}{c}
\tilde{Q}(-\epsilon) \\
\tilde{Q}^{c *}(-\epsilon)
\end{array}\right) \\
\left(\begin{array}{c}
A_{1} \cos (2 \pi M R)+B_{1} \sin (2 \pi M R) \\
C_{2} \cos (2 \pi M R)+D_{2} \sin (2 \pi M R)
\end{array}\right) & =\left(\begin{array}{cc}
\cos (2 \pi \alpha) & \sin (2 \pi \alpha) \\
-\sin (2 \pi \alpha) & \cos (2 \pi \alpha)
\end{array}\right)\left(\begin{array}{c}
A_{1} \\
-C_{2}
\end{array}\right) .
\end{aligned}
$$

Eq. (A.39) leads to a similar equation exchanging subscripts, $1 \leftrightarrow 2$. Hence we have,

$$
\begin{aligned}
& A_{1}[\cos (2 \pi M R)-\cos (2 \pi \alpha)]+B_{1} \sin (2 \pi M R)+C_{2} \sin (2 \pi \alpha)=0, \\
& C_{2}[\cos (2 \pi M R)+\cos (2 \pi \alpha)]+D_{2} \sin (2 \pi M R)+A_{1} \sin (2 \pi \alpha)=0, \\
& A_{2}[\cos (2 \pi M R)-\cos (2 \pi \alpha)]+B_{2} \sin (2 \pi M R)+C_{1} \sin (2 \pi \alpha)=0, \\
& C_{1}[\cos (2 \pi M R)+\cos (2 \pi \alpha)]+D_{1} \sin (2 \pi M R)+A_{2} \sin (2 \pi \alpha)=0 .
\end{aligned}
$$

There are enough conditions of eq. (A.41), (A.42), (A.45)-(A.48) for 8 coefficients. We solve them,

$$
\begin{aligned}
& A_{1}\left[\left(1+\xi^{2}\right) \cos (2 \pi M R)-\left(1-\xi^{2}\right) \cos (2 \pi \alpha)\right]+2 A_{2} \xi \sin (2 \pi \alpha)=0, \\
& A_{2}\left[\left(1+\xi^{2}\right) \cos (2 \pi M R)-\left(1-\xi^{2}\right) \cos (2 \pi \alpha)\right]+2 A_{1} \xi \sin (2 \pi \alpha)=0,
\end{aligned}
$$

where $\xi \equiv \frac{y_{U 5} H_{u}}{2}$. Because $A_{1}= \pm A_{2}$ is obviously a solution of eqs. (A.49), (A.50), a consistency condition is found to be

$$
\cos (2 \pi M R)=\cos (2 \pi \alpha \pm 2 \theta)
$$

where $\tan \theta \equiv \xi\left(\right.$ or $\theta=M_{t} \pi R$ ). Hence squark mass eigenvalues are given by

$$
M=\frac{n+\alpha}{R} \pm M_{t} \quad(n: \text { integer }) .
$$


For completeness, we determine coefficients. In a case of $A_{1}=A_{2}$,

$$
B_{1}=B_{2}=C_{1}=C_{2}=A_{1} \tan \left(M_{t} \pi R\right), \quad D_{1}=D_{2}=-A_{1},
$$

and in the other case of $A_{1}=-A_{2}$,

$$
B_{1}=-B_{2}=-C_{1}=C_{2}=-A_{1} \tan \left(M_{t} \pi R\right), \quad D_{1}=-D_{2}=A_{1} .
$$

The KK expansion for squark is

$$
\begin{aligned}
\tilde{Q}= & \sum_{n=-\infty}^{\infty} N_{b} \tilde{t}_{1}^{(n)}(x)\left[\cos \left(\frac{n+\alpha}{R}+M_{t}\right) y+\tan \left(M_{t} \pi R\right) \operatorname{sign}(y) \sin \left(\frac{n+\alpha}{R}+\frac{\theta}{\pi R}\right) y\right] \\
& +\sum_{n=-\infty}^{\infty} N_{b} \tilde{t}_{2}^{(n)}(x)\left[\cos \left(\frac{n+\alpha}{R}-M_{t}\right) y-\tan \left(M_{t} \pi R\right) \operatorname{sign}(y) \sin \left(\frac{n+\alpha}{R}-M_{t}\right) y\right], \\
\tilde{Q}^{c *}= & \sum_{n=-\infty}^{\infty} N_{b} \tilde{t}_{1}^{(n)}(x)\left[-\sin \left(\frac{n+\alpha}{R}+M_{t}\right) y+\tan \left(M_{t} \pi R\right) \operatorname{sign}(y) \cos \left(\frac{n+\alpha}{R}+M_{t}\right) y\right] \\
& -\sum_{n=-\infty}^{\infty} N_{b} \tilde{t}_{2}^{(n)}(x)\left[\sin \left(\frac{n+\alpha}{R}-M_{t}\right) y+\tan \left(M_{t} \pi R\right) \operatorname{sign}(y) \cos \left(\frac{n+\alpha}{R}-M_{t}\right) y\right], \\
\tilde{U}^{*}= & \sum_{n=-\infty}^{\infty} N_{b} \tilde{t}_{1}^{(n)}(x)\left[\cos \left(\frac{n+\alpha}{R}+M_{t}\right) y+\tan \left(M_{t} \pi R\right) \operatorname{sign}(y) \sin \left(\frac{n+\alpha}{R}+M_{t}\right) y\right] \\
& -\sum_{n=-\infty}^{\infty} N_{b} \tilde{t}_{2}^{(n)}(x)\left[\cos \left(\frac{n+\alpha}{R}-M_{t}\right) y-\tan \left(M_{t} \pi R\right) \operatorname{sign}(y) \sin \left(\frac{n+\alpha}{R}-M_{t}\right) y\right], \\
& +\sum_{n=-\infty}^{\infty} N_{b} \tilde{t}_{2}^{(n)}(x)\left[\sin \left(\frac{n+\alpha}{R}-M_{t}\right) y+\tan \left(M_{t} \pi R\right) \operatorname{sign}(y) \cos \left(\frac{n+\alpha}{R}-M_{t}\right) y\right], \\
\tilde{U}^{c}= & \sum_{n=-\infty}^{\infty} N_{b} \tilde{t}_{1}^{(n)}(x)\left[-\sin \left(\frac{n+\alpha}{R}+M_{t}\right) y+\tan \left(M_{t} \pi R\right) \operatorname{sign}(y) \cos \left(\frac{n+\alpha}{R}+M_{t}\right) y\right] \\
& \left.\sum^{\infty}\right)
\end{aligned}
$$

where $N_{b}=\frac{\cos \left(M_{t} \pi R\right)}{2 \pi^{1 / 2}}$ is a normalization factor.

\section{B Infinite sum}

In order to deal with infinite sums, we replace each element of a sum with a pole in a complex integral. For a function that has no singularities on the real $z$ axis, $f(k, z)$, there is a useful relation,

$$
\sum_{n=-\infty}^{\infty} f(k, n+\alpha)=\sum_{n=-\infty}^{\infty} \oint_{C_{n}^{\alpha}} d z f(k, z) \frac{\operatorname{coth}[i \pi(z-\alpha)]}{2}
$$



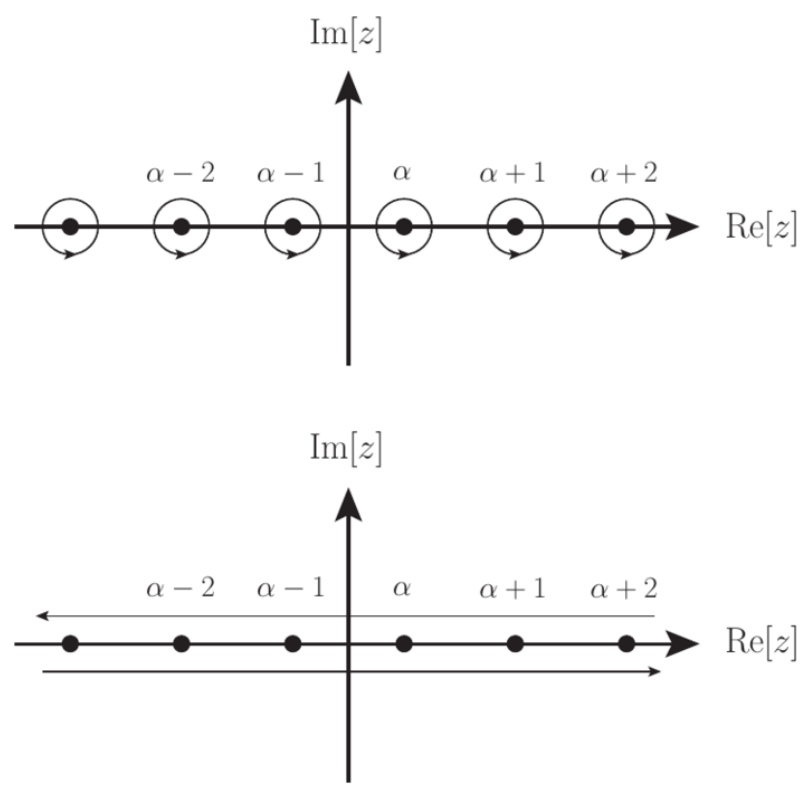

Figure 7. Integral path of $C_{n}^{\alpha}$ in the upper plot. They are combined as shown in the lower plot.

where the contour $C_{n}^{\alpha}$ is a path which rounds about $z=n+\alpha$ with an infinitesimal radius. When $z \rightarrow \alpha$,

$$
\frac{\operatorname{coth}[i \pi(z-\alpha)]}{2}=\frac{1}{2 i \pi(z-\alpha)}+\mathcal{O}(z-\alpha)
$$

and the function above is periodic under a transformation of $z \rightarrow z+n \pi$, so each $\oint_{C_{n}^{\alpha}} d z$ generates a discrete point of $f(k, z)$. We combine all the contours to paths as in figure 7 and obtain a form with a simple integral,

$$
\begin{aligned}
& \sum_{n=-\infty}^{\infty} \oint_{C_{n}^{\alpha}} d z f(k, z) \frac{\operatorname{coth}[i \pi(z-\alpha)]}{2} \\
& \quad=\left(\int_{\infty+i \epsilon}^{-\infty+i \epsilon} d z+\int_{-\infty-i \epsilon}^{\infty-i \epsilon} d z\right) f(k, z) \frac{\operatorname{coth}[i \pi(z-\alpha)]}{2} \\
& \quad=\int_{-\infty-i \epsilon}^{\infty-i \epsilon} d z\left\{f(k, z) \frac{\operatorname{coth}[i \pi(z-\alpha)]}{2}-f(k,-z) \frac{\operatorname{coth}[i \pi(-z-\alpha)]}{2}\right\} \\
& \quad=\int_{-\infty}^{\infty} d z\left\{\frac{f(k, z)+f(k,-z)}{2}\right\}+\int_{-\infty-i \epsilon}^{\infty-i \epsilon} d z\left\{\frac{f(k, z)}{e^{2 i \pi(z-\alpha)}-1}+\frac{f(k,-z)}{e^{2 i \pi(z+\alpha)}-1}\right\} .
\end{aligned}
$$

Here, we used

$$
\operatorname{coth}(x)=1+\frac{2}{e^{2 x}-1}=-\left(1+\frac{2}{e^{-2 x}-1}\right)
$$

We are interested in $f(k, z)=1 /\left(k^{2}+z^{2}\right), z /\left(k^{2}+z^{2}\right)$, and hence, for such functions that damps for $z \rightarrow \pm \infty-i \epsilon$ and can be suppressed by $e^{-2 i \pi z}$ for $z \rightarrow-i \infty$, the second expression 
of eq. (B.3) can enclose the path, referred as to $C_{\nabla}$, in the negative imaginary $z$ plane,

$$
\sum_{n=-\infty}^{\infty} f(k, n+\alpha)=\int_{-\infty}^{\infty} d z\left\{\frac{f(k, z)+f(k,-z)}{2}\right\}+\oint_{C_{\nabla}} d z\left\{\frac{f(k, z)}{e^{2 i \pi(z-\alpha)}-1}+\frac{f(k,-z)}{e^{2 i \pi(z+\alpha)}-1}\right\} .
$$

If $f(k, z)$ has poles inside the closed path $C_{\nabla}$, the second term on the right-hand side becomes a function of $k$, otherwise it vanishes. In the following section, we will see the cases of,

$$
f(k, z)=\frac{1}{k^{2}+z^{2}}, \frac{z}{k^{2}+z^{2}} .
$$

Using eq. (B.5), formulae of infinite sum are

$$
\begin{aligned}
& \sum_{n=-\infty}^{\infty} \frac{1}{k^{2}+(n+\alpha)^{2}}=\int_{-\infty}^{\infty} \frac{d z}{k^{2}+z^{2}}+\frac{\pi}{k}\left\{\frac{1}{e^{2 \pi(k-i \alpha)}-1}+\frac{1}{e^{2 \pi(k+i \alpha)}-1}\right\} \\
& \sum_{n=-\infty}^{\infty} \frac{(n+\alpha)}{k^{2}+(n+\alpha)^{2}}=(-i \pi)\left\{\frac{1}{e^{2 \pi(k-i \alpha)}-1}-\frac{1}{e^{2 \pi(k+i \alpha)}-1}\right\}
\end{aligned}
$$

This is finite because the momentum dependence is exponentially suppressed in the UV regime. The divergent part appear as the first term of eq. (B.7) which has a higher power of UV divergence. However, this divergence is insensitive to supersymmetry breaking parameter, $\alpha$, and then it completely vanishes after combining bosonic and fermionic contribution. Only finite pieces depend on $\alpha$. This is consistent with the non-local nature of supersymmetry breaking by the Scherk-Schwarz mechanism. Because fields notice supersymmetry breaking only when they travel around the extra dimension, local effects still hold supersymmetric nature leading to absence of UV divergence.

Finally, we calculate the following sum and integral for the effective potential,

$$
\begin{aligned}
W^{\prime}(\omega) & =\sum_{n=-\infty}^{\infty} \int \frac{d^{4} k}{(2 \pi)^{4}} \frac{(n+\omega)}{k^{2}+(n+\omega)^{2}} \\
& =\int \frac{d k k^{3}\left(2 \pi^{2}\right)}{(2 \pi)^{4}}(-i \pi)\left\{\frac{1}{e^{2 \pi(k-i \omega)}-1}-\frac{1}{e^{2 \pi(k+i \omega)}-1}\right\} \\
& =\frac{-3 i}{2(2 \pi)^{5}}\left[\operatorname{Li}_{4}\left(e^{2 \pi i \omega}\right)-\operatorname{Li}_{4}\left(e^{-2 \pi i \omega}\right)\right]
\end{aligned}
$$

where

$$
\int_{0}^{\infty} d k \frac{k^{n}}{e^{2 \pi(k \mp i \omega)}-1}=\frac{n !}{(2 \pi)^{n+1}} \operatorname{Li}_{n+1}\left(e^{ \pm 2 \pi i \omega}\right) .
$$

Here the divergence piece does not appear because $\omega$ independent terms are already subtracted when $W$ is constructed as in eq. (3.9).

\section{Effect of higher order terms of $\mathcal{O}\left(H_{u}^{6} R^{6}\right)$ to Higgs Mass}

Top quark wave function we solved tells that top mass is polynomial of $H_{u}$ in eq. (3.5). For our Higgs mass calculation, we use an expansion with respected $H_{u} R$ and take into account 


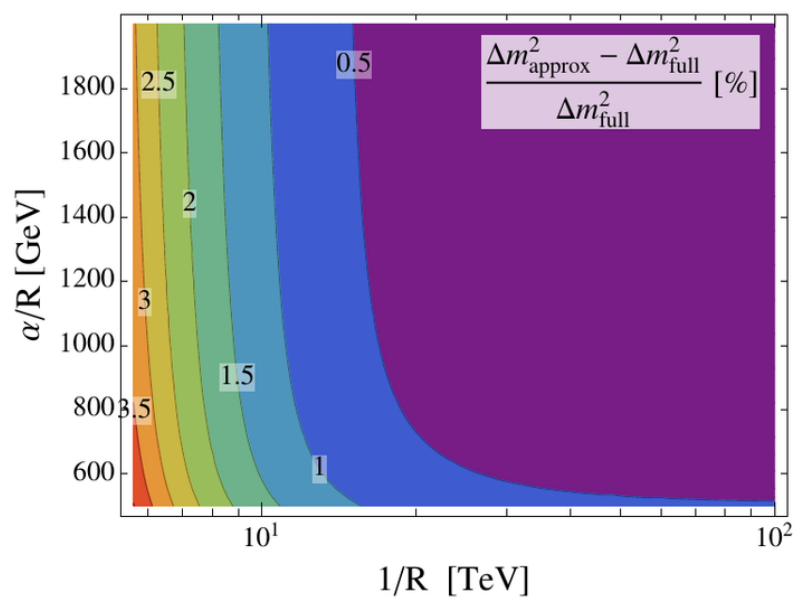

Figure 8. Deviation of $\left(\Delta m_{\text {approx }}^{2}-\Delta m^{2}\right) / \Delta m^{2}$ in percent. We fix parameters as $v_{u}=174 \mathrm{GeV}$ and $y_{t}=1$ for simplicity.

up to Higgs quartic terms as in eq. (3.16). Here we show this is good approximation in parameter of our interest, $v \ll 5 \mathrm{TeV} \lesssim R^{-1}$.

To simplify discussion, we consider the following potential depending on only $H_{u}$,

$$
V\left(H_{u}\right)=\left(\mu^{2}+m_{H_{u}}^{2}\right) H_{u}^{2}+\frac{g_{Z}^{2}}{4} H_{u}^{4}+V_{t}\left(H_{u}\right)
$$

where $g_{Z}^{2} \equiv\left(g^{2}+g^{\prime 2}\right) / 2$ and $V_{t}$ is potential from the radiative correction. The second derivative around the VEV gives the Higgs mass,

$$
m_{h}^{2} \simeq \frac{1}{2} \frac{\partial^{2} V}{\partial v_{u}^{2}}=\left(\mu^{2}+m_{H_{u}}^{2}\right)+\frac{3 g_{Z}^{2}}{2} v_{u}^{2}+\frac{1}{2} \frac{\partial^{2} V_{t}}{\partial v_{u}^{2}}=g_{Z}^{2} v_{u}^{2}+\frac{1}{2}\left(\frac{\partial^{2} V_{t}}{\partial v_{u}^{2}}-\frac{1}{v_{u}} \frac{\partial V_{t}}{\partial v_{u}}\right)
$$

where we used the vacuum condition in the last step. In our estimate we expand the potential with respect to $H_{u} R$ and take up to $H_{u}^{4}$ terms. In order to verify this approximation in parameter of our interest, $v \ll 1 / R$, we numerically evaluate the last term of eq. (C.2),

$$
\Delta m^{2} \equiv \frac{1}{2}\left(\frac{\partial^{2} V_{t}}{\partial v_{u}^{2}}-\frac{1}{v_{u}} \frac{\partial V_{t}}{\partial v_{u}}\right)
$$

In case that the full formula of $V_{t}$ without any expansion, eq. (3.13), is adopted, we refer to it as $\Delta m_{\text {full }}^{2}$. On the other hand, we denote $\Delta m_{\text {approx }}^{2}$ is $\Delta m^{2}$ using approximated $V_{t}$ that includes up to $H_{u}^{4}$ terms as in eq. (3.16). Deviation due to the approximation, $\left(\Delta m_{\text {approx }}^{2}-\Delta m_{\text {full }}^{2}\right) / \Delta m_{\text {full }}^{2}$, is shown in figure 8 , and it is at most a few percent and is less than percent for $R^{-1} \gtrsim 10 \mathrm{TeV}$. This is because the higher order terms are suppressed by $v_{u} R \ll 1$. Since this radiative correction contributes to a half of Higgs mass-squared, the Higgs mass changes only by about a quarter of the deviation, $\left(\Delta m_{\text {approx }}^{2}-\Delta m_{\text {full }}^{2}\right) / \Delta m_{\text {full }}^{2}$. Therefore the approximation of eq. (3.16) is valid, and the higher order terms of $\mathcal{O}\left(H_{u}^{6} R^{6}\right)$ can be neglected. 


\section{Threshold corrections to Higgs soft terms}

Including all the KK tower, we obtain finite results for the Higgs soft terms through 1-loop calculation,

$$
\begin{aligned}
\delta m_{H_{u}}^{2}= & \frac{N_{c} y_{t}^{2}}{16 \pi^{2}} \frac{3}{\pi^{2} R^{2}}\left[\operatorname{Li}_{3}\left(e^{2 \pi i \alpha}\right)+\operatorname{Li}_{3}\left(e^{-2 \pi i \alpha}\right)-2 \zeta(3)\right] \\
& +\frac{\sum_{A=1,2} C_{A}^{h} g_{A}^{2}}{16 \pi^{2}} \frac{-2}{\pi^{2} R^{2}}\left[\operatorname{Li}_{3}\left(e^{2 \pi i \alpha}\right)+\operatorname{Li}_{3}\left(e^{-2 \pi i \alpha}\right)-2 \zeta(3)\right], \\
\delta m_{H_{d}}^{2}= & \frac{\sum_{A=1,2} C_{A}^{h} g_{A}^{2}}{16 \pi^{2}} \frac{-2}{\pi^{2} R^{2}}\left[\operatorname{Li}_{3}\left(e^{2 \pi i \alpha}\right)+\operatorname{Li}_{3}\left(e^{-2 \pi i \alpha}\right)-2 \zeta(3)\right], \\
\delta b= & \frac{i \mu}{16 \pi^{2}} \frac{-N_{c} y_{t}^{2}+2 \sum_{A=1,2} C_{A}^{h} g_{A}^{2}}{\pi R}\left[\operatorname{Li}_{2}\left(e^{2 \pi i \alpha}\right)-\operatorname{Li}_{2}\left(e^{-2 \pi i \alpha}\right)\right] .
\end{aligned}
$$

The Casimir invariants for $\mathrm{SU}(2)_{L}$ and $\mathrm{U}(1)_{Y}$ in $\mathrm{SU}(5)$ normalization are $C_{2, \mathrm{SU}(2)_{L}}^{h}=3 / 4$ and $C_{2, \mathrm{U}(1)_{Y}}^{h}=3 / 20$. The complete diagrammatic calculations are found in ref. [84]. Since we match the theory with the MSSM at about compactification scale and solve EWSB conditions, we subtract the MSSM contributions (with the $\overline{\mathrm{DR}}$ scheme),

$$
\begin{aligned}
\delta m_{H_{u}}^{2}(Q)= & \frac{N_{c} y_{t}^{2}}{16 \pi^{2}}\left(\frac{\alpha}{R}\right)^{2}\left\{6 \log \left[\frac{Q^{2}}{(2 \pi R)^{-2}}\right]-16\right\} \\
& +\frac{\sum_{A=1,2} C_{A}^{h} g_{A}^{2}}{16 \pi^{2}}\left(\frac{\alpha}{R}\right)^{2}\left\{-4 \log \left[\frac{Q^{2}}{(2 \pi R)^{-2}}\right]+8\right\}+\mathcal{O}\left(\alpha^{4}\right), \\
\delta m_{H_{d}}^{2}(Q)= & \frac{\sum_{A=1,2} C_{A}^{h} g_{A}^{2}}{16 \pi^{2}}\left(\frac{\alpha}{R}\right)^{2}\left\{-4 \log \left[\frac{Q^{2}}{(2 \pi R)^{-2}}\right]+8\right\}+\mathcal{O}\left(\alpha^{4}\right), \\
\delta b(Q)= & \frac{N_{c} y_{t}^{2}}{16 \pi^{2}} \mu\left(\frac{\alpha}{R}\right)\left\{-2 \log \left[\frac{Q^{2}}{(2 \pi R)^{-2}}\right]+4\right\} \\
& +\frac{\sum_{A=1,2} C_{A}^{h} g_{A}^{2}}{16 \pi^{2}} \mu\left(\frac{\alpha}{R}\right)\left\{4 \log \left[\frac{Q^{2}}{(2 \pi R)^{-2}}\right]-4\right\}+\mathcal{O}\left(\alpha^{3}\right) .
\end{aligned}
$$

We check the IR effect such as $\log \alpha$ terms is certainly cancelled. And the renormalization scale we choose, $Q_{\mathrm{RG}}=\frac{1}{2 \pi R}$, the Higgs soft terms are

$$
\begin{aligned}
m_{H_{u}}^{2} & =\left(-\frac{3 y_{t}^{2}}{\pi^{2}}+\frac{3\left(g_{2}^{2}+g_{1}^{2} / 5\right)}{8 \pi^{2}}\right)\left(\frac{\alpha}{R}\right)^{2}, \\
m_{H_{d}}^{2} & =\frac{3\left(g_{2}^{2}+g_{1}^{2} / 5\right)}{8 \pi^{2}}\left(\frac{\alpha}{R}\right)^{2}, \\
b & =\left(\frac{3 y_{t}^{2}}{4 \pi^{2}}-\frac{3\left(g_{2}^{2}+g_{1}^{2} / 5\right)}{16 \pi^{2}}\right) \mu \frac{\alpha}{R} .
\end{aligned}
$$

These results are slightly different from those in ref. [19], since ref. [19] uses the cutoff regularization to matched the theories.

Open Access. This article is distributed under the terms of the Creative Commons Attribution License (CC-BY 4.0), which permits any use, distribution and reproduction in any medium, provided the original author(s) and source are credited. 


\section{References}

[1] S.P. Martin, A supersymmetry primer, Adv. Ser. Direct. High Energy Phys. 18 (1998) 1 [hep-ph/9709356] [INSPIRE].

[2] ATLAS collaboration, Observation of a new particle in the search for the Standard Model Higgs boson with the ATLAS detector at the LHC, Phys. Lett. B 716 (2012) 1 [arXiv: 1207.7214] [INSPIRE].

[3] CMS collaboration, Observation of a new boson at a mass of $125 \mathrm{GeV}$ with the CMS experiment at the LHC, Phys. Lett. B 716 (2012) 30 [arXiv:1207.7235] [InSPIRE].

[4] ATLAS, CMS collaborations, Combined Measurement of the Higgs Boson Mass in pp Collisions at $\sqrt{s}=7$ and 8 TeV with the ATLAS and CMS Experiments, Phys. Rev. Lett. 114 (2015) 191803 [arXiv:1503.07589] [INSPIRE].

[5] L.J. Hall, D. Pinner and J.T. Ruderman, A Natural SUSY Higgs Near 126 GeV, JHEP 04 (2012) 131 [arXiv:1112.2703] [InSPIRE].

[6] J.R. Espinosa and M. Quirós, On Higgs boson masses in nonminimal supersymmetric standard models, Phys. Lett. B 279 (1992) 92 [InSPIRE].

[7] Y. Nomura, D. Poland and B. Tweedie, $\mu B$-driven electroweak symmetry breaking, Phys. Lett. B 633 (2006) 573 [hep-ph/0509244] [INSPIRE].

[8] M. Dine, N. Seiberg and S. Thomas, Higgs physics as a window beyond the MSSM (BMSSM), Phys. Rev. D 76 (2007) 095004 [arXiv:0707.0005] [InSPIRE].

[9] U. Ellwanger, C. Hugonie and A.M. Teixeira, The Next-to-Minimal Supersymmetric Standard Model, Phys. Rept. 496 (2010) 1 [arXiv:0910.1785] [INSPIRE].

[10] X. Lu, H. Murayama, J.T. Ruderman and K. Tobioka, A Natural Higgs Mass in Supersymmetry from NonDecoupling Effects, Phys. Rev. Lett. 112 (2014) 191803 [arXiv: 1308.0792] [INSPIRE].

[11] P. Batra, A. Delgado, D.E. Kaplan and T.M.P. Tait, The Higgs mass bound in gauge extensions of the minimal supersymmetric standard model, JHEP 02 (2004) 043 [hep-ph/0309149] [INSPIRE].

[12] A. Maloney, A. Pierce and J.G. Wacker, D-terms, unification and the Higgs mass, JHEP 06 (2006) 034 [hep-ph/0409127] [INSPIRE].

[13] R. Harnik, G.D. Kribs, D.T. Larson and H. Murayama, The Minimal supersymmetric fat Higgs model, Phys. Rev. D 70 (2004) 015002 [hep-ph/0311349] [INSPIRE].

[14] R. Kitano and Y. Nakai, Emergent Higgs from hidden dimensions, JHEP 04 (2013) 106 [arXiv: 1212.2726] [INSPIRE].

[15] R. Kitano, M.A. Luty and Y. Nakai, Partially Composite Higgs in Supersymmetry, JHEP 08 (2012) 111 [arXiv:1206.4053] [INSPIRE].

[16] L.J. Hall, J.D. Lykken and S. Weinberg, Supergravity as the Messenger of Supersymmetry Breaking, Phys. Rev. D 27 (1983) 2359 [InSPIRE].

[17] ATLAS collaboration, Search for squarks and gluinos with the ATLAS detector in final states with jets and missing transverse momentum using $\sqrt{s}=8$ Te $V$ proton-proton collision data, JHEP 09 (2014) 176 [arXiv:1405.7875] [INSPIRE]. 
[18] T.J. LeCompte and S.P. Martin, Compressed supersymmetry after 1/fb at the Large Hadron Collider, Phys. Rev. D 85 (2012) 035023 [arXiv:1111.6897] [INSPIRE].

[19] H. Murayama, Y. Nomura, S. Shirai and K. Tobioka, Compact Supersymmetry, Phys. Rev. D 86 (2012) 115014 [arXiv:1206.4993] [INSPIRE].

[20] J. Fan, M. Reece and J.T. Ruderman, Stealth Supersymmetry, JHEP 11 (2011) 012 [arXiv:1105.5135] [INSPIRE].

[21] J. Fan, M. Reece and J.T. Ruderman, A Stealth Supersymmetry Sampler, JHEP 07 (2012) 196 [arXiv:1201.4875] [INSPIRE].

[22] R. Barbier et al., R-parity violating supersymmetry, Phys. Rept. 420 (2005) 1 [hep-ph/0406039] [INSPIRE].

[23] C. Csáki, Y. Grossman and B. Heidenreich, MFV SUSY: A Natural Theory for R-Parity Violation, Phys. Rev. D 85 (2012) 095009 [arXiv:1111.1239] [INSPIRE].

[24] J.T. Ruderman, T.R. Slatyer and N. Weiner, A Collective Breaking of R-Parity, JHEP 09 (2013) 094 [arXiv: 1207.5787] [INSPIRE].

[25] B. Bhattacherjee, J.L. Evans, M. Ibe, S. Matsumoto and T.T. Yanagida, Natural supersymmetry's last hope: R-parity violation via UDD operators, Phys. Rev. D 87 (2013) 115002 [arXiv:1301.2336] [INSPIRE].

[26] C. Csáki, E. Kuflik and T. Volansky, Dynamical R-Parity Violation, Phys. Rev. Lett. 112 (2014) 131801 [arXiv:1309.5957] [INSPIRE].

[27] J. Scherk and J.H. Schwarz, Spontaneous Breaking of Supersymmetry Through Dimensional Reduction, Phys. Lett. B 82 (1979) 60 [INSPIRE].

[28] J. Scherk and J.H. Schwarz, How to Get Masses from Extra Dimensions, Nucl. Phys. B 153 (1979) 61 [INSPIRE].

[29] R. Barbieri, L.J. Hall and Y. Nomura, A constrained standard model from a compact extra dimension, Phys. Rev. D 63 (2001) 105007 [hep-ph/0011311] [INSPIRE].

[30] I. Antoniadis, S. Dimopoulos, A. Pomarol and M. Quirós, Soft masses in theories with supersymmetry breaking by TeV compactification, Nucl. Phys. B 544 (1999) 503 [hep-ph/9810410] [INSPIRE].

[31] A. Delgado, A. Pomarol and M. Quirós, Supersymmetry and electroweak breaking from extra dimensions at the TeV scale, Phys. Rev. D 60 (1999) 095008 [hep-ph/9812489] [InSPIRE].

[32] N. Arkani-Hamed, L.J. Hall, Y. Nomura, D. Tucker-Smith and N. Weiner, Finite radiative electroweak symmetry breaking from the bulk, Nucl. Phys. B 605 (2001) 81 [hep-ph/0102090] [INSPIRE].

[33] D.M. Ghilencea and H.-P. Nilles, Quadratic divergences in Kaluza-Klein theories, Phys. Lett. B 507 (2001) 327 [hep-ph/0103151] [INSPIRE].

[34] A. Delgado, G. von Gersdorff, P. John and M. Quirós, One loop Higgs mass finiteness in supersymmetric Kaluza-Klein theories, Phys. Lett. B 517 (2001) 445 [hep-ph/0104112] [INSPIRE].

[35] R. Contino and L. Pilo, A note on regularization methods in Kaluza-Klein theories, Phys. Lett. B 523 (2001) 347 [hep-ph/0104130] [INSPIRE].

[36] R. Barbieri, L.J. Hall and Y. Nomura, Models of Scherk-Schwarz symmetry breaking in 5-D: Classification and calculability, Nucl. Phys. B 624 (2002) 63 [hep-th/0107004] [INSPIRE]. 
[37] A. Masiero, C.A. Scrucca, M. Serone and L. Silvestrini, Nonlocal symmetry breaking in Kaluza-Klein theories, Phys. Rev. Lett. 87 (2001) 251601 [hep-ph/0107201] [INSPIRE].

[38] A. Delgado, G. von Gersdorff and M. Quirós, Two loop Higgs mass in supersymmetric Kaluza-Klein theories, Nucl. Phys. B 613 (2001) 49 [hep-ph/0107233] [INSPIRE].

[39] H.D. Kim, To be (finite) or not to be, that is the question: 'Kaluza-Klein contribution to the Higgs mass', hep-ph/0106072 [INSPIRE].

[40] E.A. Mirabelli and M.E. Peskin, Transmission of supersymmetry breaking from a four-dimensional boundary, Phys. Rev. D 58 (1998) 065002 [hep-th/9712214] [INSPIRE].

[41] A. Pomarol and M. Quirós, The Standard model from extra dimensions, Phys. Lett. B 438 (1998) 255 [hep-ph/9806263] [INSPIRE].

[42] Z. Chacko and M.A. Luty, Radion mediated supersymmetry breaking, JHEP 05 (2001) 067 [hep-ph/0008103] [INSPIRE].

[43] R. Barbieri, L.J. Hall and Y. Nomura, Softly broken supersymmetric desert from orbifold compactification, Phys. Rev. D 66 (2002) 045025 [hep-ph/0106190] [INSPIRE].

[44] A. Delgado, A. Pomarol and M. Quirós, Electroweak and flavor physics in extensions of the standard model with large extra dimensions, JHEP 01 (2000) 030 [hep-ph/9911252] [INSPIRE].

[45] A. Delgado and M. Quirós, Supersymmetry and finite radiative electroweak breaking from an extra dimension, Nucl. Phys. B 607 (2001) 99 [hep-ph/0103058] [INSPIRE].

[46] R. Barbieri, G. Marandella and M. Papucci, Breaking the electroweak symmetry and supersymmetry by a compact extra dimension, Phys. Rev. D 66 (2002) 095003 [hep-ph/0205280] [INSPIRE].

[47] R. Barbieri, G. Marandella and M. Papucci, The Higgs mass as a function of the compactification scale, Nucl. Phys. B 668 (2003) 273 [hep-ph/0305044] [inSPIRE].

[48] R. Kitano and Y. Nomura, Supersymmetry, naturalness and signatures at the LHC, Phys. Rev. D 73 (2006) 095004 [hep-ph/0602096] [INSPIRE].

[49] S. Dimopoulos, K. Howe and J. March-Russell, Maximally Natural Supersymmetry, Phys. Rev. Lett. 113 (2014) 111802 [arXiv:1404.7554] [INSPIRE].

[50] G. Burdman, Z. Chacko, H.-S. Goh and R. Harnik, Folded supersymmetry and the LEP paradox, JHEP 02 (2007) 009 [hep-ph/0609152] [INSPIRE].

[51] T. Cohen, N. Craig, H.K. Lou and D. Pinner, Folded Supersymmetry with a Twist, arXiv: 1508.05396 [INSPIRE].

[52] R. Barbieri and A. Strumia, The 'LEP paradox', hep-ph/0007265 [INSPIRE].

[53] D. Marti and A. Pomarol, Supersymmetric theories with compact extra dimensions in $N=1$ superfields, Phys. Rev. D 64 (2001) 105025 [hep-th/0106256] [INSPIRE].

[54] D.E. Kaplan and N. Weiner, Radion mediated supersymmetry breaking as a Scherk-Schwarz theory, hep-ph/0108001 [INSPIRE].

[55] N. Arkani-Hamed, T. Gregoire and J.G. Wacker, Higher dimensional supersymmetry in 4 - D superspace, JHEP 03 (2002) 055 [hep-th/0101233] [INSPIRE].

[56] J.A. Bagger, F. Feruglio and F. Zwirner, Generalized symmetry breaking on orbifolds, Phys. Rev. Lett. 88 (2002) 101601 [hep-th/0107128] [INSPIRE]. 
[57] B.C. Allanach, SOFTSUSY: a program for calculating supersymmetric spectra, Comput. Phys. Commun. 143 (2002) 305 [hep-ph/0104145] [INSPIRE].

[58] H.E. Haber, R. Hempfling and A.H. Hoang, Approximating the radiatively corrected Higgs mass in the minimal supersymmetric model, Z. Phys. C 75 (1997) 539 [hep-ph/9609331] [INSPIRE].

[59] H.E. Haber and R. Hempfling, The renormalization group improved Higgs sector of the minimal supersymmetric model, Phys. Rev. D 48 (1993) 4280 [hep-ph/9307201] [InSPIRE].

[60] M. Carena, J.R. Espinosa, M. Quirós and C.E.M. Wagner, Analytical expressions for radiatively corrected Higgs masses and couplings in the MSSM, Phys. Lett. B 355 (1995) 209 [hep-ph/9504316] [INSPIRE].

[61] M. Carena, J.R. Ellis, A. Pilaftsis and C.E.M. Wagner, Renormalization group improved effective potential for the MSSM Higgs sector with explicit CP-violation, Nucl. Phys. B 586 (2000) 92 [hep-ph/0003180] [INSPIRE].

[62] S. Heinemeyer, W. Hollik and G. Weiglein, FeynHiggs: A program for the calculation of the masses of the neutral CP even Higgs bosons in the MSSM, Comput. Phys. Commun. 124 (2000) 76 [hep-ph/9812320] [INSPIRE].

[63] T. Sjöstrand, S. Mrenna and P.Z. Skands, PYTHIA 6.4 Physics and Manual, JHEP 05 (2006) 026 [hep-ph/0603175] [INSPIRE].

[64] http://www.physics.ucdavis.edu/ conway/research/software/pgs/pgs4-general.htm.

[65] https://web.physik.rwth-aachen.de/service/wiki/bin/view/Kraemer/SquarksandGluinos.

[66] W. Beenakker, R. Hopker, M. Spira and P.M. Zerwas, Squark and gluino production at hadron colliders, Nucl. Phys. B 492 (1997) 51 [hep-ph/9610490] [INSPIRE].

[67] A. Kulesza and L. Motyka, Threshold resummation for squark-antisquark and gluino-pair production at the LHC, Phys. Rev. Lett. 102 (2009) 111802 [arXiv:0807.2405] [INSPIRE].

[68] A. Kulesza and L. Motyka, Soft gluon resummation for the production of gluino-gluino and squark-antisquark pairs at the LHC, Phys. Rev. D 80 (2009) 095004 [arXiv: 0905.4749] [INSPIRE].

[69] W. Beenakker, S. Brensing, M. Krämer, A. Kulesza, E. Laenen and I. Niessen, Soft-gluon resummation for squark and gluino hadroproduction, JHEP 12 (2009) 041 [arXiv: 0909.4418] [INSPIRE].

[70] W. Beenakker et al., Squark and Gluino Hadroproduction, Int. J. Mod. Phys. A 26 (2011) 2637 [arXiv: 1105.1110] [INSPIRE].

[71] G. Bélanger, F. Boudjema, A. Pukhov and A. Semenov, MicrOMEGAs 2.0: A program to calculate the relic density of dark matter in a generic model, Comput. Phys. Commun. 176 (2007) 367 [hep-ph/0607059] [INSPIRE].

[72] G. Bélanger, F. Boudjema, A. Pukhov and A. Semenov, Dark matter direct detection rate in a generic model with MicrOMEGAs 2.2, Comput. Phys. Commun. 180 (2009) 747 [arXiv:0803.2360] [INSPIRE].

[73] Planck collaboration, P.A.R. Ade et al., Planck 2013 results. XVI. Cosmological parameters, Astron. Astrophys. 571 (2014) A16 [arXiv:1303.5076] [INSPIRE].

[74] C.G. Lester and D.J. Summers, Measuring masses of semiinvisibly decaying particles pair produced at hadron colliders, Phys. Lett. B 463 (1999) 99 [hep-ph/9906349] [INSPIRE]. 
[75] H. Murayama, M.M. Nojiri and K. Tobioka, Improved discovery of a nearly degenerate model: MUED using MT2 at the LHC, Phys. Rev. D 84 (2011) 094015 [arXiv:1107.3369] [INSPIRE].

[76] T. Appelquist, H.-C. Cheng and B.A. Dobrescu, Bounds on universal extra dimensions, Phys. Rev. D 64 (2001) 035002 [hep-ph/0012100] [InSPIRE].

[77] J. Alwall, M.-P. Le, M. Lisanti and J.G. Wacker, Model-Independent Jets plus Missing Energy Searches, Phys. Rev. D 79 (2009) 015005 [arXiv: 0809.3264] [InSPIRE].

[78] K. Rolbiecki and K. Sakurai, Constraining compressed supersymmetry using leptonic signatures, JHEP 10 (2012) 071 [arXiv:1206.6767] [INSPIRE].

[79] H.K. Dreiner, M. Krämer and J. Tattersall, How low can SUSY go? Matching, monojets and compressed spectra, Europhys. Lett. 99 (2012) 61001 [arXiv:1207.1613] [INSPIRE].

[80] H. Dreiner, M. Krämer and J. Tattersall, Exploring QCD uncertainties when setting limits on compressed supersymmetric spectra, Phys. Rev. D 87 (2013) 035006 [arXiv:1211.4981] [INSPIRE].

[81] B. Bhattacherjee, A. Choudhury, K. Ghosh and S. Poddar, Compressed supersymmetry at $14 \mathrm{TeV}$ LHC, Phys. Rev. D 89 (2014) 037702 [arXiv:1308.1526] [InSPIRE].

[82] S. Mukhopadhyay, M.M. Nojiri and T.T. Yanagida, Compressed SUSY search at the $13 \mathrm{TeV}$ LHC using kinematic correlations and structure of ISR jets, JHEP 10 (2014) 12 [arXiv: 1403.6028] [INSPIRE].

[83] C. Han and M. Park, Revealing the jet substructure in a compressed spectrum, arXiv:1507.07729 [INSPIRE].

[84] K. Tobioka, Aspects of Supersymmetry after LHC Run I, Ph.D. Thesis (2015), arXiv:1511.04099 [INSPIRE]. 